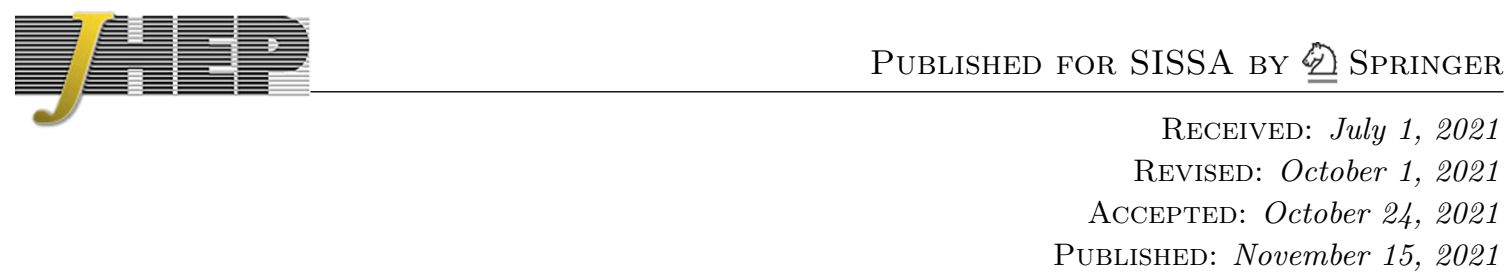

\title{
3-dimensional $\Lambda$-BMS symmetry and its deformations
}

\author{
Andrzej Borowiec, ${ }^{a}$ Jerzy Kowalski-Glikman ${ }^{a, b}$ and Josua Unger ${ }^{a}$ \\ ${ }^{a}$ Institute for Theoretical Physics, University of Wrocław, \\ pl. M. Borna 9, 50-204 Wrocław, Poland \\ ${ }^{b}$ National Centre for Nuclear Research, \\ Pasteura 7, 02-093 Warsaw, Poland \\ E-mail: andrzej.borowiec@uwr.edu.pl, \\ jerzy.kowalski-glikman@uwr.edu.pl, unger.josua@uwr.edu.pl
}

ABSTRACT: In this paper we study quantum group deformations of the infinite dimensional symmetry algebra of asymptotically AdS spacetimes in three dimensions. Building on previous results in the finite dimensional subalgebras we classify all possible Lie bialgebra structures and for selected examples we explicitely construct the related Hopf algebras. Using cohomological arguments we show that this construction can always be performed by a so-called twist deformation. The resulting structures can be compared to the wellknown $\kappa$-Poincaré Hopf algebras constructed on the finite dimensional Poincaré or (anti) de Sitter algebra. The dual $\kappa$ Minkowski spacetime is supposed to describe a specific noncommutative geometry. Importantly, we find that some incarnations of the $\kappa$-Poincaré can not be extended consistently to the infinite dimensional algebras. Furthermore, certain deformations can have potential physical applications if subalgebras are considered. Since the conserved charges associated with asymptotic symmetries in 3-dimensional form a centrally extended algebra we also discuss briefly deformations of such algebras. The presence of the full symmetry algebra might have observable consequences that could be used to rule out these deformations.

KEYwords: Quantum Groups, Models of Quantum Gravity, Non-Commutative Geometry ArXiv EPrint: 2106.12874 


\section{Contents}

1 Introduction 1

2 Asymptotic symmetries of spacetimes with cosmological constant 3

2.1 Asymptotic symmetries in 3D 3

2.2 Real forms 5

2.3 Algebra of surface charges 6

$\begin{array}{lll}3 & \text { Lie bialgebras and deformation } & 7\end{array}$

3.1 Cohomology 8

4 Twist deformation and classification $\quad 9$

$\begin{array}{ll}4.1 & \text { Twisting of the coalgebra sector } \\ \end{array}$

$\begin{array}{lll}\text { 4.1.1 Abelian twist } & 12\end{array}$

$\begin{array}{lll}\text { 4.1.2 Jordanian twist } & 12\end{array}$

$\begin{array}{lll}4.2 & \text { Contraction limit and uniqueness of deformations } & 16\end{array}$

$\begin{array}{lll}4.3 & \text { Deformations of the surface charge algebra } 20\end{array}$

5 One-sided Witt algebra and specialization 21

6 Conclusion 23

A Proof of the cohomology theorems $\quad 25$

$\begin{array}{lll}\text { A.1 Proof of theorem } 1 & 25\end{array}$

$\begin{array}{lll}\text { A.2 Proof of theorem } 2 & 29\end{array}$

B Classification of triangular r-matrices $\quad 31$

\section{Introduction}

Gravity in 3 dimensions [1-3], see [4] for review, is a remarkably rich and interesting theory. It remained relatively obscured for many years, but from the seminal paper of Witten [5] (see also [6]) it became one of the most studied theory in theoretical physics.

There are many reasons for that. Gravity in 3 dimensions is a topological field theory with no local degrees of freedom, which makes the quantum theory exactly soluble, so it can serve as a toy model of quantum gravity. As it was shown in [7] in the case of negative cosmological constant this theory possesses an asymptotic Virasoro symmetry. This result was a precursor of AdS/CFT [8] and the $\mathrm{AdS}_{3} / \mathrm{CFT}_{2}$ correspondence is actively and intensively investigated $[9,10]$. Second, in spite of having no local dynamical degrees of freedom, 3 dimensional gravity with negative cosmological constant admits black hole 
solution [11, 12], which makes 3-dimensional gravity a nice toy model for studying Hawking radiation. See [13] for a review of these aspects of the theory.

Another interesting property of 3-dimensional gravity is the fact that it provides a model of emergence of quantum group symmetries as physical symmetries of quantized gravitating systems. Envisioned in [14] and based on mathematical works [15-17] this idea was developed further, among others, in canonical formulation in [18-21], and for gravitational path integral in $[22,23]$. This aspect of gravity in 3 dimensions will be the central subject of the studies presented in this paper.

In any spacetime dimension Poincaré group and (Anti) de Sitter group are symmetries of classical vacuum spacetimes of gravity with zero, (negative) positive cosmological constant. More than half a century ago it was realized however that there are circumstances that the symmetry of particular configurations of gravitational field is much larger. It was shown that the symmetries of the asymptotically flat gravitational field near null infinity form an infinite dimensional group, called the Bondi, Metzner, Sachs (BMS) group [24-26], which contains the Poincaré group as its subgroup. A natural question then arises what are the quantum deformations of the BMS group. We addressed this problem in the case of zero cosmological constant in the recent papers [27, 28].

In these papers we found a class of quantum deformations of BMS algebra. ${ }^{1}$ Technically, we started with the twist deformation of Poincaré subalgebras of BMS algebra, extending them to the whole of BMS and obtaining in this way a Hopf-BMS algebra. Here we want to extend this analysis to the case of non-vanishing cosmological constant.

In this paper we consider deformations of BMS algebra of 3-dimensional gravity model of non-zero cosmological constant $\Lambda$-BMS algebra.

We are interested here in 3 dimensional models because, as mentioned above, it was shown using different approaches that deformed symmetries play an important role in 3 dimensional gravity. In fact, it was established in [30] using the non-perturbative methods of Loop Quantum Gravity that the symmetries of the Euclidean Anti de Sitter quantum spacetime in 3 spacetime dimensions is described by a quantum group. More precisely, that paper considers the algebra of the six conserved gravitational charges that form the Euclidean Anti de Sitter algebra. It is then shown by explicit computation that upon quantization these charges become operators, forming the $S_{q}(3,1)$ algebra, with the deformation parameter $q=\exp (i \hbar G \sqrt{|\Lambda|} / 2)$. In the $\Lambda \rightarrow 0$ limit this algebra contracts to the 3 dimensional (Euclidean) version of $\kappa$-Poincaré algebra [31-34]. It is believed that an analogous result holds in the case of Lorentzian AdS. This result is a major motivation for investigating deformations BMS algebra with infinite number of charges in 3-dimensional asymptotically AdS spacetime. Indeed if the Anti de Sitter subalgebra of these charges is getting deformed by non-perturabative quantum gravity effects it can be expected that by the same token the whole algebra of asymptotic charges is deformed as well. It is of interest therefore to list and investigate in some details a class of quantum deformations of the 3-dimensional BMS algebra in the case of negative cosmological constant.

\footnotetext{
${ }^{1}$ To be more precise, we were dealing with what is sometimes called the extended BMS which includes superrotations in contrast to the original BMS. Some authors also considered a further variant, the "generalized" BMS [29].
} 
On the more technical side the undertaking to investigate a class of deformations of AdS-BMS algebras is further motivated by the recently obtained complete classification of deformations of (Anti) de Sitter algebras in three dimensions [35] (the discussion of the corresponding contractions $\Lambda \rightarrow 0$ can be found in [40]). Using this classification in the present paper we find a class of deformations of 3-dimensional $\Lambda$-BMS algebra.

Another reason why we choose to investigate here the simpler 3 -dimensional $\Lambda$-BMS algebra is that in 4 dimensions the non-vanishing cosmological constant extension of the BMS algebra cannot have a structure of Lie algebra. More precisely, it has been recently shown in [41], by applying cohomological arguments, that there does not exist a $\Lambda$-BMS Lie algebra containing the 4 dimensional (Anti) de Sitter subalgebra that gives the $\mathrm{BMS}_{4}$ algebra as a contraction limit $\Lambda \rightarrow 0$. In fact, such extension of the $\mathrm{BMS}_{4}$ algebra should presumably have the structure of Lie algebroids, with structure functions instead of structure constants [41, 43, 44]. As a consequence to deform it we would need a theory of quantized Lie algebroids, which is much less developed than the theory of quantized enveloping algebras we are dealing with.

Note that the deformations discussed in this work deform the coalgebra structure. Deformations and (central) extensions of the algebra sector have been addressed for example in [41] for the $\mathfrak{B}_{4}$ and in [42] for diffeomorphisms on the two-sphere.

The plan of the paper is as follows. In the next section we briefly recall the structure of asymptotic symmetries $\Lambda-\mathrm{BMS}_{3}$ algebra in 3 dimensions and interpret the algebras for different signs of cosmological constant as two real forms of a complex algebra. In section 3 we discuss Lie bialgebras and deformations, first in general terms and then in the specific case of interest of two copies of Witt algebra $\Lambda-\mathrm{BMS}_{3} \simeq \mathfrak{W} \oplus \mathfrak{W}$. Section 4 is devoted to discussion of twist deformations, their classifications and contractions. We conclude our paper with some remarks on one-sided Witt algebra and specialization in section 5 .

\section{Asymptotic symmetries of spacetimes with cosmological constant}

In this section we describe the structure of the $\Lambda-\mathrm{BMS}_{3}$ algebra of asymptotic symmetries. An extensive discussion of this algebra can be found in [45] and [46], which contain also references to other works.

\subsection{Asymptotic symmetries in 3D}

The study of asymptotic symmetries is usually carried out by starting from a general metric with given asymptotic structure (usually asymptotically Minkowski or (Anti) de Sitter) and imposing fall-off conditions on the expansion coefficients close to the asymptotic boundary. Then one looks for vector fields preserving the form of the asymptotic expansion. In the three dimensional asymptotically AdS spacetime such vector fields have the form $\xi_{f, R}=f \partial_{u}+R \partial_{z}$ where $R=R(z), f=T(z)+u \partial_{z} R$ and their algebra reads $[45,46]$

$$
\begin{aligned}
{\left[\xi_{1}, \xi_{2}\right] } & =\hat{\xi} \equiv \hat{f} \partial_{u}+\hat{R} \partial_{z} \\
\hat{f} & =R_{1} \partial_{z} f_{2}+f_{1} \partial_{z} R_{2}-(1 \leftrightarrow 2), \quad \hat{R}=R_{1} \partial_{z} R_{2}-\Lambda f_{1} \partial_{z} f_{2}-(1 \leftrightarrow 2) .
\end{aligned}
$$


Parametrizing $f_{m} \equiv T_{m}=z^{m+1}$ and $R_{m} \equiv l_{m}=z^{m+1}$ we find

$$
\begin{aligned}
{\left[l_{m}, l_{n}\right] } & =(m-n) l_{m+n}, \quad\left[l_{m}, T_{n}\right]=(m-n) T_{m+n}, \\
{\left[T_{m}, T_{n}\right] } & =-\Lambda(m-n) l_{m+n}
\end{aligned}
$$

which in the contraction limit $\Lambda \rightarrow 0$ gives the usual $\mathrm{BMS}_{3}$ algebra (2.3). Depending on the sign of $\Lambda$ (2.3)-(2.4) describes two different real algebras, into which one can embed the finite $3 \mathrm{D}$ (Anti) de Sitter algebra ${ }^{2}$ generated by the generators $K_{2}, K_{ \pm}, M_{+-}, M_{ \pm 2}$ satisfying the algebra

$$
\begin{aligned}
{\left[K_{+}, K_{-}\right] } & =-\eta_{+-} \Lambda M_{+-}, & {\left[K_{ \pm}, K_{2}\right] } & =-\eta_{+-} \Lambda M_{ \pm 2} \\
{\left[M_{+2}, M_{-2}\right] } & =-\eta_{22} M_{+-}, & {\left[M_{+-}, M_{ \pm 2}\right] } & = \pm \eta_{+-} M_{ \pm 2}, \quad\left[M_{+-}, K_{ \pm}\right]= \pm \eta_{+-} K_{ \pm} \\
{\left[M_{ \pm 2}, K_{2}\right] } & =\eta_{22} K_{ \pm}, & {\left[M_{ \pm 2}, K_{\mp}\right] } & =-\eta_{+-} K_{2}
\end{aligned}
$$

in an infinitely many distinct ways by identifying (we rescale $\Lambda \rightarrow n^{2} \Lambda$ )

$$
K_{2}=\frac{T_{0}}{n}, \quad K_{ \pm}=-\frac{T_{ \pm n}}{\sqrt{2} n}, \quad M_{+-}=\frac{l_{0}}{n}, \quad M_{ \pm 2}=\mp \frac{1}{\sqrt{2} n} l_{ \pm n}
$$

Furthermore, the $\Lambda$-BMS 3 algebra (2.3)-(2.4) is isomorphic to two copies of the Witt algebra $\mathfrak{W} \oplus \mathfrak{W}$ via

$$
\begin{aligned}
L_{m} & =\frac{1}{2}\left(l_{m}+\frac{1}{\sqrt{-\Lambda}} T_{m}\right), & \bar{L}_{m} & =\frac{1}{2}\left(l_{m}-\frac{1}{\sqrt{-\Lambda}} T_{m}\right) \\
\Rightarrow\left[L_{m}, L_{n}\right] & =(m-n) L_{m+n}, & {\left[\bar{L}_{m}, \bar{L}_{n}\right] } & =(m-n) \bar{L}_{m+n}, \quad\left[L_{m}, \bar{L}_{n}\right]=0 .
\end{aligned}
$$

The isomorphism (2.9) is complex for positive $\Lambda$ and therefore (2.10) has to be seen as a complex algebra with different real forms (cf. next section). As before, it is also easy to see from (2.9) that there are infinitely many embeddings of the (A)dS algebra into the $\Lambda$-BMS 3 , i.e. one shows that the $\mathfrak{o}(4, \mathbb{C}) \equiv \mathfrak{s l}(2, \mathbb{C}) \oplus \mathfrak{s l}(2, \mathbb{C})$ is multiply embedded in the two copies of the Witt algebra via

$$
\begin{aligned}
& L_{0}, L_{ \pm n}, \bar{L}_{0}, \bar{L}_{ \pm n}, \\
& L_{n} \rightarrow \frac{L_{n}}{n}, \quad \bar{L}_{n} \rightarrow \frac{\bar{L}_{n}}{n}, \quad n=1,2, \ldots .
\end{aligned}
$$

Using the isomorphism (2.9) this translates to the family of embeddings

$$
l_{0}, l_{ \pm n}, T_{0}, T_{ \pm n}
$$

with the rescaling

$$
l_{n} \rightarrow \frac{l_{n}}{n}, \quad \sqrt{-\Lambda} \rightarrow n \sqrt{-\Lambda}, \quad n=1,2, \ldots
$$

Alternatively one can rescale $\eta_{\mu \nu}$ instead of the generators $l_{m}$, i.e. instead of (2.8) one would have the same relations with $n=1$ and $\Lambda$ is not rescaled.

\footnotetext{
${ }^{2}$ AdS corresponds to $\Lambda<0$ and dS to $\Lambda>0$ and we choose the metric to be $\eta_{+-}=1, \eta_{22}=-1$. From the point of view of four-dimensional geometry one can set $\eta_{33}=-\eta_{+-} \Lambda$ and $K_{ \pm}=M_{ \pm 3}, K_{2}=M_{23}$.
} 


\section{$2.2 \quad$ Real forms}

A real Lie algebra is naturally defined as a real vector space with Lie bracket determined by real structure constants. However, for the purpose of quantum deformation one needs another, equivalent definition, which is based on the notion of a real form of a complex Lie algebra (see e.g. [35] and references therein). Thus real form is a pair $(\mathfrak{g}, \dagger)$ where $\mathfrak{g}$ is a complex Lie algebra and $\dagger: \mathfrak{g} \rightarrow \mathfrak{g}$ denotes antilinear involutive antiautomorphism mimicking Hermitian conjugation, see below. If the structure constants are real then the natural choice is $X_{a}^{\dagger}=-X_{a}, X_{a} \in \operatorname{gen}(\mathfrak{g})$. Favorite physicist convention is to establish imaginary structure constants and Hermitian generators $Y_{a}^{\dagger}=Y_{a}$, where $Y_{a}=i X_{a}$.

For example, the simple $\mathfrak{s l}(2, \mathbb{C})$ Lie algebra admits (up to an isomorphism) two real forms: noncompact $\mathfrak{s l}(2, \mathbb{R}) \sim \mathfrak{o}(1,2) \sim \mathfrak{s u}(1,1)$ and compact $\mathfrak{o}(3) \sim \mathfrak{s u}(2) .{ }^{3}$ Accordingly, the semisimple $\mathfrak{o}(4, \mathbb{C})=\mathfrak{s l}(2, \mathbb{C}) \oplus \mathfrak{s l}(2, \mathbb{C})$ admits four non-isomorphic real forms: Euclidean, Lorentzian, Kleinian and quaternionic [37,38]. Each of them can be extended to the real form of the infinite-dimensional $\Lambda$-BMS algebra. However, in view of possible physical applications we are interested here in Lorentzian and Kleinian type. They correspond to de Sitter and anti de Sitter algebras of 3 -dimensional Lorentzian spacetime $\mathbb{R}^{1,2}$.

Note that while (2.5)-(2.7) describes two different real algebras with $\Lambda \lessgtr 0$, in (2.10) there is only one complex algebra with two different reality conditions. If we consider the subalgebra $\mathfrak{o}(4, \mathbb{C})$ spanned by $L_{0}, L_{ \pm 1}, \bar{L}_{0}, \bar{L}_{ \pm 1}$

$$
\begin{array}{ll}
{\left[L_{0}, L_{ \pm 1}\right]=\mp L_{ \pm 1},} & {\left[L_{+1}, L_{-1}\right]=2 L_{0},} \\
{\left[\bar{L}_{0}, \bar{L}_{ \pm 1}\right]=\mp \bar{L}_{ \pm 1},} & {\left[\bar{L}_{+1}, \bar{L}_{-1}\right]=2 \bar{L}_{0},}
\end{array}
$$

it is related to the standard Cartan-Weyl form

$$
\begin{array}{ll}
{\left[H, E_{ \pm}\right]= \pm E_{ \pm},} & {\left[E_{+}, E_{-}\right]=2 H} \\
{\left[\bar{H}, \bar{E}_{ \pm}\right]= \pm \bar{E}_{ \pm}, \quad\left[\bar{E}_{+}, \bar{E}_{-}\right]=2 \bar{H}}
\end{array}
$$

$\operatorname{via}^{4}$

$$
H=-L_{0}, \quad E_{ \pm}=i L_{ \pm 1}, \quad \bar{H}=-\bar{L}_{0}, \quad \bar{E}_{ \pm}=i \bar{L}_{ \pm 1} .
$$

In (2.15) there are two real forms that correspond to the AdS and dS case resprectively. For negative $\Lambda$, i.e. the AdS case we have from (2.9) that

$$
\begin{aligned}
L_{m}^{\dagger}=-L_{m}, & \bar{L}_{m}^{\dagger}=-\bar{L}_{m} \\
\Leftrightarrow H^{\dagger}=-H, \quad E_{ \pm}^{\dagger}=E_{ \pm}, & \bar{H}^{\dagger}=-\bar{H}, \quad \bar{E}_{ \pm}^{\dagger}=\bar{E}_{ \pm}
\end{aligned}
$$

and restrained to the subalgebra this defines two copies of the real form $\mathfrak{s l}(2, \mathbb{R}) \simeq \mathfrak{o}(2,1)$. Thus this real form corresponds to the Kleinian algebra $\mathfrak{o}(2,2) \simeq \mathfrak{o}(2,1) \oplus \overline{\mathfrak{o}}(2,1)$.

\footnotetext{
${ }^{3}$ Different notational coventions reference to different $\star$ realisations or different system of generators.

${ }^{4}$ It is worth noticing that Cartan-Weyl generators of $\mathfrak{s l}(2, \mathbb{R})$ can be also considered as light-cone generators of $\mathfrak{o}(1,2)$ through the identification: $M_{+-}=H, M_{1 \pm}=E_{ \pm}$with non-diagonal metric components $\eta_{+-}=\eta_{-+}=1$ and diagonal one $\eta_{22}=-2$ (cf. (2.5)).
} 
The other case with positive $\Lambda$ yields

$$
\begin{aligned}
L_{m}^{\ddagger} & =-\bar{L}_{m}, \quad \bar{L}_{m}^{\ddagger}=-L_{m}, \\
\Leftrightarrow H^{\ddagger} & =-\bar{H}, \quad E_{ \pm}^{\ddagger}=\bar{E}_{ \pm},
\end{aligned}
$$

i.e. the Lorentzian real form when restricted to the $\mathfrak{o}(4, \mathbb{C})$ subalgebra. This can be identified with the real structures listed in [35] in the last line of eq. (4.13) and eq. (4.14) with the automorphism $E_{ \pm} \rightarrow-E_{ \pm}, \bar{E}_{ \pm} \rightarrow-\bar{E}_{ \pm}$. Note that this automorphism of the $\mathfrak{s l}(2, \mathbb{C})$

$$
\Phi\left(E_{ \pm}\right)=-E_{ \pm}, \quad \Phi(H)=H, \rightarrow \Phi\left(L_{ \pm 1}\right)=-L_{ \pm 1}, \quad \Phi\left(L_{0}\right)=L_{0}
$$

can be extended uniquely to an automorphism of the Witt algebra.

As mentioned above for the algebras (2.5)-(2.7) we have only one reality condition

$$
K_{\mu}^{\dagger}=K_{\mu}, \quad M_{\mu \nu}^{\dagger}=M_{\mu \nu}
$$

\subsection{Algebra of surface charges}

By the Noether theorem the new-found symmetries correspond to conserved quantities, although this relation is more involved in the case of gauge symmetries (see [45]). For example in the flat case one obtains infinitely many charges parametrized by supertranslations $f(z, \bar{z})[39]$

$$
Q[f]=\frac{1}{4 \pi G} \int d^{2} z \gamma_{z \bar{z}} f m_{B}
$$

where the integral is defined over a boundary of a spacelike slice and $m_{B}$ is the Bondi mass aspect. These charges generate the symmetry transformations and are thus tightly linked to the algebra derived above. In fact the Poisson bracket of the charges has to coincide with the Lie bracket of the algebra generators up to a constant, i.e. it is in general a central extension of the algebra [45]. For the Witt algebra it is well known that the central extension is uniquely given by the Virasoro algebra ( $\mathfrak{V i r})$ satisfying the commutation relations

$$
\left[L_{m}, L_{n}\right]=(m-n) L_{m+n}+\frac{c_{L}}{12}\left(m^{3}-m\right) \delta_{m+n, 0} .
$$

Furthermore, two copies of the Virasoro algebra consitute the only possibility for a central extension of $\mathfrak{W} \oplus \mathfrak{W}$ and Brown and Henneaux calculated in their seminal paper [7] that

$$
c_{L}=c_{\bar{L}}=\frac{3}{2 G \sqrt{-\Lambda}}
$$

It should be noticed that only the first embedding $\left(L_{0}, L_{ \pm 1}\right)$ of $\mathfrak{s l}(2)$ algebra is not affected by the presence of central charge in (2.27). The higher order embeddings, e.g. $\left(L_{0}, L_{ \pm 2}\right)$, have to take into account the central charge. 


\section{Lie bialgebras and deformation}

Recall that a Lie bialgebra is a Lie algebra $\mathfrak{g}$ with a cobracket $\delta: \mathfrak{g} \rightarrow \mathfrak{g} \otimes \mathfrak{g}$ satisfying the cocycle condition [47]

$$
\delta([x, y])=[x \otimes 1+1 \otimes x, \delta(y)]-[y \otimes 1+1 \otimes y, \delta(x)]
$$

and the dual version of the Jacobi identity, the so-called co-Jacobi identity

$$
\operatorname{Cycl}((\delta \otimes \mathrm{id})) \delta(x)=0
$$

with $\operatorname{Cycl}(a \otimes b \otimes c)=a \otimes b \otimes c+c \otimes a \otimes b+b \otimes c \otimes a$.

A coboundary Lie bialgebra has a cobracket defined by a classical r-matrix $r \in \bigwedge^{2} \mathfrak{g}$ via

$$
\delta_{r}(x)=[x \otimes 1+1 \otimes x, r]
$$

and $\delta_{r}$ satisfies the co-Jacobi identity iff $r=a \wedge b$ fulfills the modified classical Yang-Baxter equation

$$
[[r, r]] \equiv\left[r_{12}, r_{13}\right]+\left[r_{12}, r_{23}\right]+\left[r_{13}, r_{23}\right]=\Omega
$$

where $r_{12}=a \otimes b \otimes 1-b \otimes a \otimes 1$ and $\Omega$ has to be ad-invariant in $\mathfrak{g}$. If the r.h.s. of (3.4) vanishes the Lie bialgebra is called triangular.

A $*$-Lie bialgebra over a real form of a complex algebra with an involution $*$ is a Lie bialgebra that is a $*$ vector space and bracket and cobracket are $*$ homomorphisms. The latter condition implies for coboundary Lie bialgebras defined by an r-matrix $r$ that

$$
r^{* \otimes *}=-r
$$

where $(a \otimes b)^{* \otimes *}:=a^{*} \otimes b^{*}$.

We recall that two $r$-matrices $r_{1}, r_{2} \in \mathfrak{g} \wedge \mathfrak{g} \subset \mathfrak{g} \otimes \mathfrak{g}$ are called equivalent if there exists a Lie algebra automorphism $\phi \in \operatorname{Aut}(\mathfrak{g})$ such that $(\phi \otimes \phi)\left(r_{1}\right)=r_{2}$. Equivalent $r$ matrices provide isomorphic Lie bialgebra structures on $\mathfrak{g}$. Choosing Lie subalgebras $\mathfrak{h} \subset \mathfrak{g}$ and $r_{1}, r_{2} \in \mathfrak{h} \wedge \mathfrak{h} \subset \mathfrak{g} \wedge \mathfrak{g}$ one can ask now whether $\mathfrak{h}$-equivalence implies $\mathfrak{g}$-equivalence. The answer is not obvious since in general an automorphism of $\mathfrak{h}$ does not extend to the automorphism of the full algebra $\mathfrak{g}$. Therefore, the classification problem depends on the choice of an algebra we are interested in, instead of just the minimal subalgebra generated by the $r$-matrix itself. ${ }^{5}$ Similarly, if $(\mathfrak{g}, \star)$ is a real form of a complex Lie algebra $\mathfrak{g}$ then $\operatorname{Aut}(\mathfrak{g}, \star) \subset \operatorname{Aut}(\mathfrak{g})$. Therefore, equivalent complex $r$-matrices may not be equivalent as real ones.

Lie bialgebras can be considered as infinitesimal versions of Hopf algebras, i.e. unitary algebras with a compatible coproduct $\Delta$, counit $\varepsilon$ and an antipode $S$ generalizing the

\footnotetext{
${ }^{5}$ This problem involves only triangular case. Non-triangular $r$-matrices can not be promoted from subalgebras, cf. (3.4).
} 
inverse (cf. [47, 48] for an extensive treatment of Hopf algebras/quantum groups). In particular the cobracket is related to the coproduct via

$$
\delta=\lim _{\kappa \rightarrow \infty} \frac{\Delta-\Delta^{\mathrm{op}}}{1 / \kappa},
$$

where $1 / \kappa$ is the deformation parameter (see below). Starting from any Lie algebra $\mathfrak{g}$ one can generically construct a Hopf algebra $H$ by considering the universal enveloping algebra $U \mathfrak{g}$ with

$$
\Delta_{0}(x)=x \otimes 1+1 \otimes x, \quad \varepsilon(x)=0, \quad S_{0}(x)=-x .
$$

Non-trivial coalgebra structures can be obtained by a deformation, i.e. $U \mathfrak{g}$ is first topologically extended to $U \mathfrak{g}[[1 / \kappa]]$ with the so-called $h$-adic topology to include formal power series in the deformation parameter $1 / \kappa$. If $\mathfrak{g}$ admits a triangular Lie bialgebra structure such a deformation can be obtained by a twisting procedure, i.e. then a twist $\mathcal{F} \in H \otimes H$ satisfying the 2-cocycle condition

$$
\mathcal{F}_{12}\left(\Delta_{0} \otimes 1\right)(\mathcal{F})=\mathcal{F}_{23}\left(1 \otimes \Delta_{0}\right)(\mathcal{F})
$$

exists and defines a deformed coproduct via

$$
\Delta_{\mathcal{F}}=\mathcal{F} \Delta_{0} \mathcal{F}^{-1}
$$

In the following we will find that all possible deformations are of this form in the $\mathfrak{W} \oplus \mathfrak{W}$ algebra with the help of Lie algebra cohomology.

\subsection{Cohomology}

It is well known that the relation

$$
\delta\left(\left[L_{m}, L_{n}\right]\right)=\left[L_{m} \otimes 1+1 \otimes L_{m}, \delta\left(L_{n}\right)\right]-\left[L_{n} \otimes 1+1 \otimes L_{n}, \delta\left(L_{m}\right)\right]
$$

is the condition that $\delta$ is a 1-cocycle of the Chevalley-Eilenberg cohomology [49]. Recall that this cohomology is constructed on the vector spaces of cochains $C^{n}=\operatorname{Hom}\left(\Lambda^{n} \mathfrak{g}, V\right)$ where $V$ is a module of the Lie algebra $\mathfrak{g}$ (in our case $V=\Lambda^{2} \mathfrak{g}$ ). The coboundary operators $\partial_{n}: C^{n} \rightarrow C^{n+1}$ are given by

$$
\begin{aligned}
\partial_{n}(f)\left(x_{1} \wedge \ldots \wedge x_{n}+1\right)= & \sum_{i=1}^{n+1}(-1)^{i} x_{i} \triangleright f\left(x_{1} \wedge \ldots \wedge \hat{x}_{i} \ldots \wedge x_{n+1}\right) \\
& +\sum_{i<j}(-1)^{i+j} f\left(\left[x_{i}, x_{j}\right] \wedge x_{1} \ldots \wedge \hat{x}_{i} \ldots \wedge \hat{x}_{j} \ldots \wedge x_{n+1}\right)
\end{aligned}
$$

where $\hat{x}_{i}$ means that the $i$-th tensor leg is dropped and $\triangleright$ denotes the right action on the module. We denote the cohomology groups by Ker $\partial_{n} / \operatorname{Im} \partial_{n-1} \equiv H^{n}(\mathfrak{g}, V)$.

If the first cohomology $H^{1}\left(\mathfrak{W} \oplus \mathfrak{W}, \bigwedge^{2}(\mathfrak{W} \oplus \mathfrak{W})\right)$ vanishes it would follow that all Lie bialgebras in $\mathfrak{W} \oplus \mathfrak{W}$ are coboundary. The result is even a bit stronger as not all cocycles of 
the cohomology define Lie bialgebras but only those which additionally fulfill the co-Jacobi identity.

In the theory of finite dimensional Lie algebras a fundamental result is the Whitehead lemma which states that all cohomology groups $H^{n}(\mathfrak{g}, V)$ for finite-dimensional semi-simple $\mathfrak{g}$ and $V$ vanish. However, since $\mathfrak{W}$ is not finite dimensional the lemma is not applicable here. Thus we prove the following theorem in appendix A

Theorem 1 The first cohomology $H^{1}\left(\mathfrak{W}, \bigwedge^{2} \mathfrak{W}\right)$ of the Witt algebra with values in the exterior product of the adjoint module is zero.

Using this theorem one can also prove the following

Theorem 2 The first cohomology $H^{1}\left(\mathfrak{W} \oplus \mathfrak{W}, \bigwedge^{2}(\mathfrak{W} \oplus \mathfrak{W})\right)$ is zero.

In [56] the authors independently prove a slightly more general result than our first theorem at the cost of a longer proof. The proofs presented here conceptually follow the proof of $H^{1}(\mathfrak{W}, \mathfrak{W})=\{0\}$ in $[50]$.

As stated above the second theorem establishes that all Lie bialgebra structures are coboundary and we now just need to show that the corresponding deformations are all given by a twist.

\section{Twist deformation and classification}

In the recent paper [28] several twists were considered for the BMS algebra in three and four dimensions (denoted by $\mathfrak{B}_{3}$ and $\mathfrak{B}_{4}$, respectively). It was noticed that all deformations from coboundary Lie bialgebras have to be triangular since there is no ad-invariant element in $\wedge^{3} \mathfrak{B}_{3}$ and $\wedge^{3} \mathfrak{B}_{4}$. The same observation also holds for $\mathfrak{W} \oplus \mathfrak{W}$. Since in four dimensions the $\Lambda$-BMS is a Lie algebroid it is not known how a suitable concept of quantum group can be defined on it. In three dimensions, however, we can investigate the possible twists in a similar way.

As motivated earlier we will focus on $\Lambda<0$ in the following, if not stated otherwise. In the contraction limit one can identify the generators $K_{i}$ with the momenta of the $\mathfrak{B}_{3}$, i.e.

$$
\lim _{\Lambda \rightarrow 0} K_{i}=P_{i}
$$

Let us first consider the three dimensional Poincare $\left(\mathfrak{P}_{3}\right)$. The abelian twist and the Jordanian twist, corresponding to the r-matrices

$$
r_{J}=i \eta^{+-} M_{+-} \wedge P_{+}, \quad r_{A}=-i \eta^{+-} M_{+-} \wedge P_{2}
$$

are then also viable if $P_{i}$ is replaced with $K_{i}$, i.e. the r-matrices are triangular and the twists satisfy the 2-cocycle condition. Also the r-matrix associated with the light-cone $\kappa$-Poincaré

$$
r_{\mathrm{LC}}=i M_{+-} \wedge K_{+}-i M_{+2} \wedge K_{2}
$$


is triangular in three dimensions and when expressed in terms of $L_{m}, \bar{L}_{m}$

$$
r_{\mathrm{LC}}=-\frac{i \Lambda \sqrt{2}}{n}\left(L_{0} \wedge L_{n}-\bar{L}_{0} \wedge \bar{L}_{n}\right)
$$

and it is apparent that it coincides with $r_{I I}(\zeta=0)$ from [35] and [40] where all classes of available twists of $\mathfrak{o}(4)$ were obtained. Demanding triangularity we are left with the following r-matrices from the classification

$$
\begin{aligned}
r_{I} & =\chi\left(E_{+}-\bar{E}_{+}\right) \wedge(H+\bar{H}), \\
r_{I I} & =\chi E_{+} \wedge H+\bar{\chi} \bar{E}_{+} \wedge \bar{H}+\zeta E_{+} \wedge \bar{E}_{+}, \\
r_{I I I} & =\eta H \wedge \bar{H} \\
r_{V} & =\bar{\chi} \bar{E}_{+} \wedge \bar{H}+\rho H \wedge \bar{E}_{+} .
\end{aligned}
$$

The abelian twist corresponds to $r_{I I I}$ and the Jordanian twist to $r_{I}$.

In general there are also other classical r-matrices in $\mathfrak{W} \oplus \mathfrak{W}$ and the full classification is not known even for the Witt algebra [36]. For example it is easy to see that r-matrices of the form

$$
r=\left(\sum_{i} \alpha_{i} L_{i}\right) \wedge \bar{L}_{m}
$$

are triangular.

However, one has to take into account that the asymptotic symmetry is spontaneously broken in the bulk in the sense that the vacua related by supertranslations and superrotations are physically distinguishable [39]. There is a correspondence between these vacua and the embeddings of Poincaré subalgebras which leave the associated vacuum invariant. Therefore we require that the restriction of the Hopf algebra deformed by the twist associated with a given r-matrix to an embedding is a sub Hopf algebra and we are interested in r-matrices of the form (4.5)-(4.8) where $\left\{H, E_{ \pm}, \bar{H}, \bar{E}_{ \pm}\right\}$is replaced with the embedding. Note that while in the case of positive $\Lambda$ the involution mixes left and right-handed elements this is not happening for negative $\Lambda$, leaving the potential possibility to use different embeddings for them.

The classification (4.5)-(4.8) is defined up to automorphisms of the $\mathfrak{o}(4)$ but there might be inequivalent r-matrices that are related by $\operatorname{Aut}(\mathfrak{o}(4))$ that do not extend to $\operatorname{Aut}(\mathfrak{W} \oplus \mathfrak{W})$.

Therefore, in appendix B the classification of triangular r-matrices on $\mathfrak{o}(4)$ is revised along the lines of $[37,38]$ but using only the $\mathfrak{W} \oplus \mathfrak{W}$ automorphisms $(\operatorname{Aut}(\mathfrak{W} \oplus \mathfrak{W})$ )

$$
\begin{aligned}
\varphi_{(\gamma, \bar{\gamma}, \epsilon, \bar{\epsilon})}\left(L_{m}\right) & =\gamma^{m} \epsilon L_{\epsilon m}, & \varphi_{(\gamma, \bar{\gamma}, \epsilon, \bar{\epsilon})}\left(\bar{L}_{m}\right) & =\bar{\gamma}^{m} \bar{\epsilon}_{\bar{\epsilon}} \bar{L}_{\bar{\epsilon} m}, \\
\varphi^{\prime}\left(L_{m}\right) & =\bar{L}_{m}, & \varphi^{\prime}\left(\bar{L}_{m}\right) & =L_{m},
\end{aligned}
$$


where $0 \neq \gamma, \bar{\gamma} \in \mathbb{C}, \epsilon, \bar{\epsilon}= \pm 1$. As a result we obtain the following classes of r-matrices

$$
\begin{aligned}
& r_{1^{\prime}}=\beta\left(L_{1}+L_{-1}+2 L_{0}\right) \wedge\left(\bar{L}_{1}+\bar{L}_{-1}+2 \bar{L}_{0}\right)+a_{1}+\bar{a}_{1}, \\
& r_{2^{\prime}}=\beta L_{1} \wedge \bar{L}_{0}+a_{2}, \\
& r_{3^{\prime}}=\beta\left(L_{1}+\epsilon L_{0}+\epsilon^{\prime} L_{-1}\right) \wedge\left(\bar{L}_{1}+\bar{L}_{-1}+2 \bar{L}_{0}\right)+\bar{a}_{1}+(1-\epsilon)\left(1-\epsilon^{\prime}\right) a_{2}, \\
& r_{4^{\prime}}=\beta_{1} L_{1} \wedge \bar{L}_{1}+\beta_{2}\left(L_{1}+\bar{L}_{1}\right) \wedge\left(L_{0}+\bar{L}_{0}\right), \\
& r_{5^{\prime}}=\beta\left(L_{1}+L_{-1}\right) \wedge\left(\bar{L}_{1}+\bar{L}_{0}\right)+a_{1}, \\
& r_{6^{\prime}}=\left(\beta L_{1}+\beta_{0} L_{0}+\epsilon \beta L_{-1}\right) \wedge\left(\bar{\beta} \bar{L}_{1}+\bar{\beta}_{0} \bar{L}_{0}+\bar{\epsilon} \bar{\beta} \bar{L}_{-1}\right), \\
& r_{7^{\prime}}=L_{1} \wedge\left(\bar{\beta} \bar{L}_{1}+\bar{\beta} \bar{L}_{0}+\epsilon \bar{\beta} \bar{L}_{-1}\right)+a_{2}, \\
& r_{8^{\prime}}=\beta L_{1} \wedge \bar{L}_{1}+a_{2}+\bar{a}_{2},
\end{aligned}
$$

where $\epsilon, \epsilon^{\prime}, \bar{\epsilon} \in\{0,1\}$ and

$$
\begin{array}{ll}
a_{1}=\alpha\left(L_{1} \wedge L_{0}+L_{1} \wedge L_{-1}-L_{-1} \wedge L_{0}\right), & a_{2}=\alpha L_{1} \wedge L_{0}, \\
\bar{a}_{1}=\bar{\alpha}\left(\bar{L}_{1} \wedge \bar{L}_{0}+\bar{L}_{1} \wedge \bar{L}_{-1}-\bar{L}_{-1} \wedge \bar{L}_{0}\right), & \bar{a}_{2}=\bar{\alpha} \bar{L}_{1} \wedge \bar{L}_{0} .
\end{array}
$$

In the case of complex $\mathfrak{W} \oplus \mathfrak{W}$ all the parameters in (4.12)-(4.19) can take values in $\mathbb{C}$ but for the real forms associated with the involutions $\dagger, \ddagger$ the condition (3.5) constrains the choice of parameters. For $\dagger$ in the classes $r_{1^{\prime}}$ to $r_{5^{\prime}}$ and $r_{7^{\prime}}, r_{8^{\prime}}$ this enforces $\beta, \beta_{1}, \beta_{2}, \alpha, \bar{\alpha} \in$ $i \mathbb{R}$ and in $r_{6^{\prime}}$ one can restrict $\beta, \beta_{0} \in i \mathbb{R}$ and $\bar{\beta}, \bar{\beta}_{0} \in \mathbb{R}$ without loss of generality.

For positive $\Lambda$, i.e. the involution $\ddagger$, the reality condition is more restrictive. In particular

$$
\begin{aligned}
& r_{1^{\prime}}: \quad \alpha=\bar{\alpha} \in i \mathbb{R}, \beta \in \mathbb{R}, \quad r_{2^{\prime}}: \quad \beta=0, \alpha=\bar{\alpha} \in i \mathbb{R}, \\
& r_{3^{\prime}}: \quad \bar{\alpha}=0, \epsilon=\epsilon^{\prime}=1, \beta \in \mathbb{R}, \quad r_{4^{\prime}}: \quad \beta_{1} \in \mathbb{R}, \beta_{2} \in i \mathbb{R}, \\
& r_{5^{\prime}} \text { : excluded, } \quad r_{6^{\prime}}: \quad \epsilon=\bar{\epsilon}, \beta_{0} \bar{\beta}=-\frac{\beta^{*}}{\beta_{0}^{*}} \text { or }\left(\beta_{0}=\bar{\beta}_{0}=0, \beta, \bar{\beta} \in \mathbb{R}\right), \\
& r_{7^{\prime}}: \text { excluded, } \quad r_{8^{\prime}}: \quad \beta \in \mathbb{R}, \alpha=\bar{\alpha} \in i \mathbb{R} \text {. }
\end{aligned}
$$

As the r-matrices from (4.12)-(4.19) that are not included in (4.5)-(4.8) are at least in $\mathfrak{o}(4)$ automorphism orbits containing them one can use the inverse of the automorphisms to obtain the full twists. For example $r=\left(L_{1}-L_{-1}\right) \wedge\left(\bar{L}_{1}-\bar{L}_{-1}\right)$ (which is automorphic to $r_{6^{\prime}}$ with $\left.\beta_{0}=\bar{\beta}_{0}=0, \epsilon=\bar{\epsilon}=1\right)$ is automorphic to $L_{0} \wedge \bar{L}_{0} \hat{=} r_{I I I}$ by

$$
\varphi\left(L_{1}\right)=-\frac{1}{2}\left(L_{1}+L_{-1}\right)+L_{0}, \quad \varphi\left(L_{-1}\right)=-\frac{1}{2}\left(L_{1}+L_{-1}\right)-L_{0}, \quad \varphi\left(L_{0}\right)=\frac{1}{2}\left(L_{1}-L_{-1}\right) .
$$

From the abelian twist for $r_{I I I}$

$$
\mathcal{F}_{I I I}=\exp \left(\eta L_{0} \wedge \bar{L}_{0}\right)
$$

one then gets the twist

$$
\mathcal{F}=\left(\varphi^{-1} \otimes \varphi^{-1}\right) \mathcal{F}_{I I I}=\exp \left(\eta\left(L_{1}-L_{-1}\right) \wedge\left(\bar{L}_{1}-\bar{L}_{-1}\right)\right) .
$$




\subsection{Twisting of the coalgebra sector}

In this section we will explicitly construct the Hopf algebras from an abelian and a Jordanian twist. The abelian twist here has the peculiarity that it consists only of elements that are contained in all embeddings so it does not single out any specific. The Jordanian twist can already be constructed in a very basic example namely the only non-abelian two dimensional algebra

$$
[X, Y]=Y,
$$

where $\mathcal{F}=\exp (X \otimes \log (1+Y))$ satisfies the 2-cocycle condition (3.8). Because of the semisimplicity of the relevant algebras here there is always a Cartan element that diagonalizes the adjoint action and thus a subalgebra of the form (4.25). Indeed many of the possible twists are of this form or have it as a building block, making it an ideal example to study.

\subsubsection{Abelian twist}

The abelian twist can be expressed as

$$
\mathcal{F}_{A}=\exp \left(-\frac{i}{\kappa n^{2}} \Lambda \bar{L}_{0} \wedge L_{0}\right) \exp \left(-\frac{i}{\kappa n^{2}} \Lambda\left(L_{0} \otimes L_{0}-\bar{L}_{0} \otimes \bar{L}_{0}\right)\right)
$$

which factorizes into the twist $\mathcal{F}_{3^{\prime \prime}}$ from [35] and a factor that only produces symmetric deformations of the coproduct. Explicitly

$$
\begin{aligned}
& \Delta_{\mathcal{F}_{3^{\prime \prime}}}\left(L_{m}\right)=e^{i \frac{m}{n^{2}} \Lambda \bar{L}_{0}} \otimes L_{m}+L_{m} \otimes e^{-i \frac{m}{n^{2}} \Lambda \bar{L}_{0}} \\
& \Delta_{\mathcal{F}_{3^{\prime \prime}}}\left(\bar{L}_{m}\right)=e^{-i \frac{m}{n^{2}} \Lambda L_{0}} \otimes \bar{L}_{m}+\bar{L}_{m} \otimes e^{i \frac{m}{n^{2}} \Lambda L_{0}}
\end{aligned}
$$

and

$$
\begin{aligned}
\Delta_{\mathcal{F}_{A}}\left(L_{m}\right) & =e^{\frac{i}{\kappa} \frac{m}{n^{2}} \Lambda\left(\bar{L}_{0}+L_{0}\right)} \otimes L_{m}+L_{m} \otimes e^{-\frac{i}{\kappa} \frac{m}{n^{2}} \Lambda\left(\bar{L}_{0}-L_{0}\right)} \\
\Delta_{\mathcal{F}_{A}}\left(\bar{L}_{m}\right) & =e^{-\frac{i}{\kappa} \frac{m}{n^{2}} \Lambda\left(\bar{L}_{0}+L_{0}\right)} \otimes \bar{L}_{m}+\bar{L}_{m} \otimes e^{\frac{i}{\kappa} \frac{m}{n^{2}} \Lambda\left(L_{0}-\bar{L}_{0}\right)} \\
\Delta_{\mathcal{F}_{A}}\left(L_{0}\right) & =L_{0} \otimes 1+1 \otimes L_{0}, \quad \Delta_{\mathcal{F}_{A}}\left(\bar{L}_{0}\right)=\bar{L}_{0} \otimes 1+1 \otimes \bar{L}_{0} .
\end{aligned}
$$

The antipodes can also be inferred easily from

$$
m \circ(S \otimes \mathrm{id}) \circ \Delta=1 \circ \epsilon
$$

and turn out to be

$$
\begin{array}{ll}
S_{\mathcal{F}_{3^{\prime \prime}}}\left(L_{m}\right)=-L_{m}, & S_{\mathcal{F}_{3^{\prime \prime}}}\left(\bar{L}_{m}\right)=-\bar{L}_{m}, \\
S_{\mathcal{F}_{A}}\left(L_{m}\right)=-L_{m} e^{\frac{2 i}{\kappa} \frac{m}{n^{2}} \Lambda L_{0}}, & S_{\mathcal{F}_{A}}\left(\bar{L}_{m}\right)=-\bar{L}_{m} e^{-\frac{2 i}{\kappa} \frac{m}{n^{2}} \Lambda \bar{L}_{0}}
\end{array}
$$

\subsubsection{Jordanian twist}

Considering the Jordanian twist

$$
\mathcal{F}_{J, n}=\exp \left(-\frac{1}{n}\left(L_{0}+\bar{L}_{0}\right) \otimes \log \left(1-\frac{i \Lambda}{\kappa \sqrt{2}}\left(L_{n}-\bar{L}_{n}\right)\right)\right)
$$


one finds

$$
\begin{aligned}
\Delta_{\mathcal{F}_{J, n}}\left(L_{0}\right) & =L_{0} \otimes 1+1 \otimes L_{0}-\frac{1}{n}\left(L_{0}+\bar{L}_{0}\right) \otimes \frac{d \sigma_{n}}{d\left(L_{n}-\bar{L}_{n}\right)} n L_{n} \\
& =L_{0} \otimes 1+1 \otimes L_{0}-\tilde{a}\left(L_{0}+\bar{L}_{0}\right) \otimes L_{n} \Pi_{+n}^{-1}, \\
\Delta_{\mathcal{F}_{J, n}}\left(\bar{L}_{0}\right) & =\bar{L}_{0} \otimes 1+1 \otimes \bar{L}_{0}+\tilde{a}\left(L_{0}+\bar{L}_{0}\right) \otimes \bar{L}_{n} \Pi_{+n}^{-1},
\end{aligned}
$$

where

$$
\sigma_{n} \equiv \log \left(1+\tilde{a}\left(L_{n}-\bar{L}_{n}\right)\right), \quad \Pi_{+n}=e^{\sigma_{n}}, \quad \tilde{a} \equiv \frac{-i \Lambda}{\kappa \sqrt{2}}
$$

Using (4.32) and the previous equations we find

$$
\begin{aligned}
S_{\mathcal{F}_{J, n}}\left(L_{0}\right) & =-\left(L_{0}+\tilde{a}\left(\bar{L}_{0} L_{n}+L_{0} L_{n}\right)\right) \frac{\Pi_{+n}^{-1}}{1-\Pi_{+n}^{-1}\left(L_{n}-\bar{L}_{n}\right)} \\
& =-\left(L_{0}+\tilde{a}\left(\bar{L}_{0} L_{n}+L_{0} L_{n}\right)\right), \\
S_{\mathcal{F}_{J, n}}\left(\bar{L}_{0}\right) & =-\left(\bar{L}_{0}-\tilde{a}\left(\bar{L}_{0} \bar{L}_{n}+L_{0} \bar{L}_{n}\right)\right) .
\end{aligned}
$$

For general generators we find

$$
\begin{aligned}
\Delta_{\mathcal{F}_{J, n}}\left(L_{m}\right) & =\mathcal{F}_{J, n}\left(L_{m} \otimes 1+1 \otimes L_{m}\right) \mathcal{F}_{J, n}^{-1} \\
& =L_{m} \otimes \Pi_{+n}^{\frac{m}{n}}+\sum_{l=0}^{\infty} \frac{1}{l !}\left(\frac{L_{0}+\bar{L}_{0}}{n}\right)^{l} \otimes\left[\sigma_{n},\left[\ldots,\left[\sigma_{n}, L_{m}\right] \ldots\right]\right.
\end{aligned}
$$

with

$$
\begin{aligned}
{\left[\sigma_{n}, L_{m}\right] } & =\left[-\sum_{j=0}^{\infty} \frac{(-\tilde{a})^{j}}{j}\left(L_{n}-\bar{L}_{n}\right)^{j}, L_{m}\right] \\
{\left[\left(L_{n}-\bar{L}_{n}\right)^{j}, L_{m}\right] } & =\sum_{s=0}^{j-1}\left(L_{n}-\bar{L}_{n}\right)^{s}(n-m) L_{m+n}\left(L_{n}-\bar{L}_{n}\right)^{j-s} \\
& =\sum_{k=1}^{j}\left(\sum_{s_{1}=k-1}^{j-1} \ldots \sum_{s_{k}=0}^{s_{k-1}-1} L_{m+k n}\left(L_{n}-\bar{L}_{n}\right)^{j-k} \prod_{p=0}^{k-1}(n-(m+p n))\right)
\end{aligned}
$$

where in the last line we iteratively commuted the $s$ terms in front of $L_{m+k n}$ to the right.

From this formula it can be seen that in general there is an infinite number of terms in the coproduct involving a tower of infinitely many different generators. However, when restricting ourself to (two copies of) the one-sided Witt algebra spanned by $\left\{L_{m}, \bar{L}_{m} ; m \leq\right.$ $1\}$ the situation is different. In that case there are only two possible embeddings with $n= \pm 1$ and by choosing $n=1$ the sum over $k$ in (4.42) terminates after $\min \{1-m, j\}$ terms instead of when $k=j$ which is not finite as we sum $j$ to infinity. As a consequence also the sum over $l$ terminates after $1-m$ terms and there appear only a finite number of generators and as we will see also only a finite number of terms. 
In that case we proceed with the identity

$$
\sum_{s_{1}=k-1}^{j-1} \cdots \sum_{s_{k}=0}^{s_{k-1}-1}=\frac{j !}{(j-k) ! k !}=\left(\begin{array}{l}
j \\
k
\end{array}\right)
$$

It is straightforward to see that it holds for $k=1$. Now assume that it holds for $k=k^{\prime}, 1 \leq$ $k^{\prime}<j$ and it follows that the l.h.s. for $k=k^{\prime}+1$ is

$$
\sum_{s_{1}^{\prime}=k^{\prime}}^{j-1} \sum_{s_{2}^{\prime}=k^{\prime}-1}^{s_{1}^{\prime}-1} \ldots \sum_{s_{k^{\prime}}^{\prime}=0}^{s_{k^{\prime}-1}^{\prime}-1}=\sum_{s_{1}^{\prime}=k^{\prime}}^{j-1}\left(\begin{array}{l}
s_{1}^{\prime} \\
k^{\prime}
\end{array}\right)=\left(\begin{array}{c}
j \\
k^{\prime}+1
\end{array}\right)
$$

where in the last step the hockey stick identity was used. Thus we proved (4.44) by induction. Therefore

$$
\begin{aligned}
{\left[\sigma_{1}, L_{m}\right] } & =-\sum_{j=0}^{\infty} \frac{(-\tilde{a})^{j}}{j}\left[\left(L_{n}-\bar{L}_{n}\right)^{j}, L_{m}\right] \\
& =L_{m}-\sum_{j=1}^{\infty} \sum_{k=1}^{\min \{j, 1-m\}} \frac{(-\tilde{a})^{j}}{j}\left(\begin{array}{l}
j \\
k
\end{array}\right) L_{m+k}\left(L_{1}-\bar{L}_{1}\right)^{j-k} \prod_{p=0}^{k-1}(1-(m+p))
\end{aligned}
$$

and comparing with

$$
\frac{d^{k} \sigma_{1}}{d L_{1}^{k}}=-\sum_{j=k}^{\infty} \frac{(-\tilde{a})^{j}}{j} \frac{j !}{(j-k) !}\left(L_{1}-\bar{L}_{1}\right)^{j-k}
$$

one finds that the summands in (4.46) and

$$
L_{m}+\sum_{k=1}^{1-m} L_{m+k} \frac{d^{k} \sigma_{1}}{d L_{1}^{k}} \frac{1}{k !} \prod_{p=0}^{k-1}(1-(m+p))
$$

are identical. Splitting the sums in (4.46) and (4.48) according to

$$
\begin{array}{r}
\left(\sum_{j=1}^{1-m} \sum_{k=1}^{j}+\sum_{j=2-m}^{\infty} \sum_{k=1}^{1-m}\right) \ldots \\
\left(\sum_{k=1}^{1-m} \sum_{j=k+1}^{1-m}+\sum_{k=1}^{1-m} \sum_{j=2-m}^{\infty}\right) \ldots
\end{array}
$$

and using

$$
\sum_{j=1}^{1-m} \sum_{k=1}^{j} \ldots=\sum_{k=1}^{1-m} \sum_{j=k}^{1-m} \ldots
$$


it follows that (4.42) is indeed given by (4.48) if the one-sided Witt algebra with $n=1$ is considered. Plugging the result into (4.41) yields

$$
\begin{aligned}
\Delta_{\mathcal{F}_{J, 1}}\left(L_{m}\right)= & L_{m} \otimes \Pi_{+}^{m}+\sum_{l=1}^{1-m} \frac{1}{l !}\left(\sum_{k_{1}=0}^{1-m} \frac{1}{k !} \prod_{p_{1}=0}^{k_{1}-1}\left(1-\left(m+p_{1}\right)\right)\right. \\
& \times\left(\sum_{k_{2}=0}^{1-\left(m+k_{1}\right)} \frac{1}{k_{2} !} \prod_{p_{2}=0}^{k_{2}-1}\left(1-\left(m+k_{1}+p_{2}\right)\right)\right. \\
& \times\left(\ldots\left(\sum_{k_{l}=0}^{1-\left(m+k_{1}+\ldots k_{l-1}\right)} \frac{1}{k_{l} !} \prod_{p_{l}=0}^{k_{l}-1}\left(1-\left(m+k_{1}+\ldots+k_{l-1}+p_{l}\right)\right) A \otimes B\right) \ldots\right),
\end{aligned}
$$

where

$$
\begin{aligned}
A \otimes B & =\left(\frac{L_{0}+\bar{L}_{0}}{n}\right)^{l} \otimes L_{m+k_{1}+\ldots+k_{l}} \frac{d^{k_{1}} \sigma_{1}}{d L_{1}^{k_{1}}} \ldots \frac{d^{k_{l}} \sigma_{1}}{d L_{1}^{k_{l}}} \\
\frac{d^{k} \sigma_{1}}{d L_{1}^{k}} & =-(-\tilde{a})^{k} \Pi_{+}^{-k} k !
\end{aligned}
$$

and all sums are finite for $m \leq 1$. Similarly for $\bar{L}_{m}$ one finds

$$
\begin{aligned}
\Delta_{\mathcal{F}_{J, 1}}\left(\bar{L}_{m}\right)= & \bar{L}_{m} \otimes \Pi_{+}^{m}+\sum_{l=1}^{1-m} \frac{1}{l !}\left(\sum_{k_{1}=0}^{1-m} \frac{1}{k !} \prod_{p_{1}=0}^{k_{1}-1}\left(1-\left(m+p_{1}\right)\right)\right. \\
& \times\left(\sum_{k_{2}=0}^{1-\left(m+k_{1}\right)} \frac{1}{k_{2} !} \prod_{p_{2}=0}^{k_{2}-1}\left(1-\left(m+k_{1}+p_{2}\right)\right)\right. \\
& \times\left(\ldots\left(\sum_{k_{l}=0}^{1-\left(m+k_{1}+\ldots k_{l-1}\right)} \frac{1}{k_{l} !} \prod_{p_{l}=0}^{k_{l}-1}\left(1-\left(m+k_{1}+\ldots+k_{l-1}+p_{l}\right)\right) \bar{A} \otimes \bar{B}\right) \ldots\right)
\end{aligned}
$$

where

$$
\bar{A} \otimes \bar{B}=\left(\frac{L_{0}+\bar{L}_{0}}{n}\right)^{l} \otimes \bar{L}_{m+k_{1}+\ldots+k_{l}} \frac{d^{k_{1}} \sigma_{1}}{d \bar{L}_{1}^{k_{1}}} \ldots \frac{d^{k_{l}} \sigma_{1}}{d \bar{L}_{1}^{k_{l}}} .
$$

Then the antipodes follow from (4.32) and read

$$
\begin{aligned}
S_{\mathcal{F}_{\mathrm{LC}}}\left(L_{m}\right)= & -\sum_{l=1}^{1-m} \frac{1}{l !}\left(\sum_{k_{1}=0}^{1-m} \frac{1}{k !} \prod_{p_{1}=0}^{k_{1}-1}\left(1-\left(m+p_{1}\right)\right)\right. \\
& \times\left(\sum_{k_{2}=0}^{1-\left(m+k_{1}\right)} \frac{1}{k_{2} !} \prod_{p_{2}=0}^{k_{2}-1}\left(1-\left(m+k_{1}+p_{2}\right)\right)\right. \\
& \times\left(\ldots\left(\sum_{k_{l}=0}^{1-\left(m+k_{1}+\ldots k_{l-1}\right)} \frac{1}{k_{l} !} \prod_{p_{l}=0}^{k_{l}-1}\left(1-\left(m+k_{1}+\ldots+k_{l-1}+p_{l}\right)\right) S(A) \otimes B\right) \ldots\right) \Pi_{+}^{-m},
\end{aligned}
$$


and $S(A)$ can be inferred from (4.39)

$$
S(A)=S\left(\left(L_{0}+\bar{L}_{0}\right)^{l}\right)=\left(-\left(L_{0}+\bar{L}_{0}\right) \Pi_{+}\right)^{l} .
$$

\subsection{Contraction limit and uniqueness of deformations}

So far we obtained general Hopf algebras on the symmetry algebras for asymptotically AdS spacetimes which algebraically also carry over to the dS case easily. There are several reasons why the asymptotically flat case is of special interest though. One motivation is the possibility of deformed dispersion relations that is associated with non-trivial Hopf algebra structures but in (A)dS there are no true momenta. However, by performing the contraction limit $\Lambda \rightarrow 0$, one can obtain information about quantum groups in the three dimensional BMS from the $\Lambda$-BMS. For all r-matrices of the $\mathfrak{o}(4)$ the contraction limit was obtained in [40]. The resulting r-matrices were compared to the full classification of r-matrices of the Poincaré algebra in three dimensions from [51] and it was claimed that all of them could be derived by an appropriate contraction limit. It should be noted that the contraction is ambiguous, i.e. the contraction of a class of r-matrices can be performed in different non-equivalent ways (cf. below) and not injective, i.e. there are r-matrices in the 3D Poincaré that can be obtained as a contraction from distinct $\mathfrak{o}(4) \mathrm{r}$-matrices. The general scheme relating different classes of r-matrices and their contractions is depicted in figure 1.

Let us first explicitly perform the contraction limit of $r_{1}^{\prime}$ from (4.12). To this end it is expressed in terms of $l_{m}, T_{m}$ with the help of (2.9) and subsequently expanded in powers of $1 / \sqrt{-\Lambda}$

$$
\begin{aligned}
r_{1^{\prime}}= & \frac{\alpha+\bar{\alpha}}{-\Lambda}\left(T_{1} \wedge T_{0}-T_{1} \wedge T_{-1}-T_{-1} \wedge T_{0}\right)+ \\
& \frac{\beta}{\sqrt{-\Lambda}}\left(l_{1}+l_{-1}+2 l_{0}\right) \wedge\left(T_{1}+T_{-1}+2 T_{0}\right)+\frac{\alpha}{\sqrt{-\Lambda}}(\ldots)+\beta(\ldots) .
\end{aligned}
$$

In order to obtain a finite result we have to rescale $(\alpha+\bar{\alpha}) \rightarrow(\hat{\alpha}+\hat{\bar{\alpha}})(-\Lambda)$ and $\beta \rightarrow \hat{\beta} \sqrt{-\Lambda}$. Then taking the limit $\Lambda \rightarrow 0$ yields

$$
\hat{r}_{1^{\prime}, a}=(\hat{\alpha}+\hat{\bar{\alpha}})\left(T_{1} \wedge T_{0}+T_{1} \wedge T_{-1}-T_{-1} \wedge T_{0}\right)+\hat{\beta}\left(l_{1}+l_{-1}+2 l_{0}\right) \wedge\left(T_{1}+T_{-1}+2 T_{0}\right) .
$$

This is not the only possibility to abtain a finite limit, in the case $\alpha=-\bar{\alpha}$ one can also rescale $\alpha \rightarrow \hat{\alpha} \sqrt{-\Lambda}$ to get

$$
\begin{aligned}
\hat{r}_{1^{\prime}, b}= & \beta\left(l_{1} \wedge T_{1}+l_{-1} \wedge T_{-1}+4 l_{0} \wedge T_{0}\right)+(2 \beta+\alpha)\left(l_{1} \wedge T_{0}+l_{0} \wedge T_{-1}\right) \\
& +(2 \beta-\alpha)\left(l_{0} \wedge T_{1}+l_{-1} \wedge T_{0}\right)+(\beta+\alpha) l_{1} \wedge T_{-1}+(\beta-\alpha) l_{-1} \wedge T_{1} .
\end{aligned}
$$

Similarly the contraction limit can be performed for all r-matrices in (4.12)-(4.19). Comparing to (the triangular part of) the classification of r-matrices on $\mathfrak{P}_{3}$ by [51] shows that the contractions of $r_{1^{\prime}}$ are in general not automorphic to it via a $\mathfrak{B}_{3}$ automorphism. Instead the set of r-matrices of the $\mathfrak{P}_{3}$ up to $\operatorname{Aut}\left(\mathfrak{B}_{3}\right)$ is strictly larger than up to $\operatorname{Aut}\left(\mathfrak{P}_{3}\right)$ similar to the case of non-vanishing cosmological constant. 


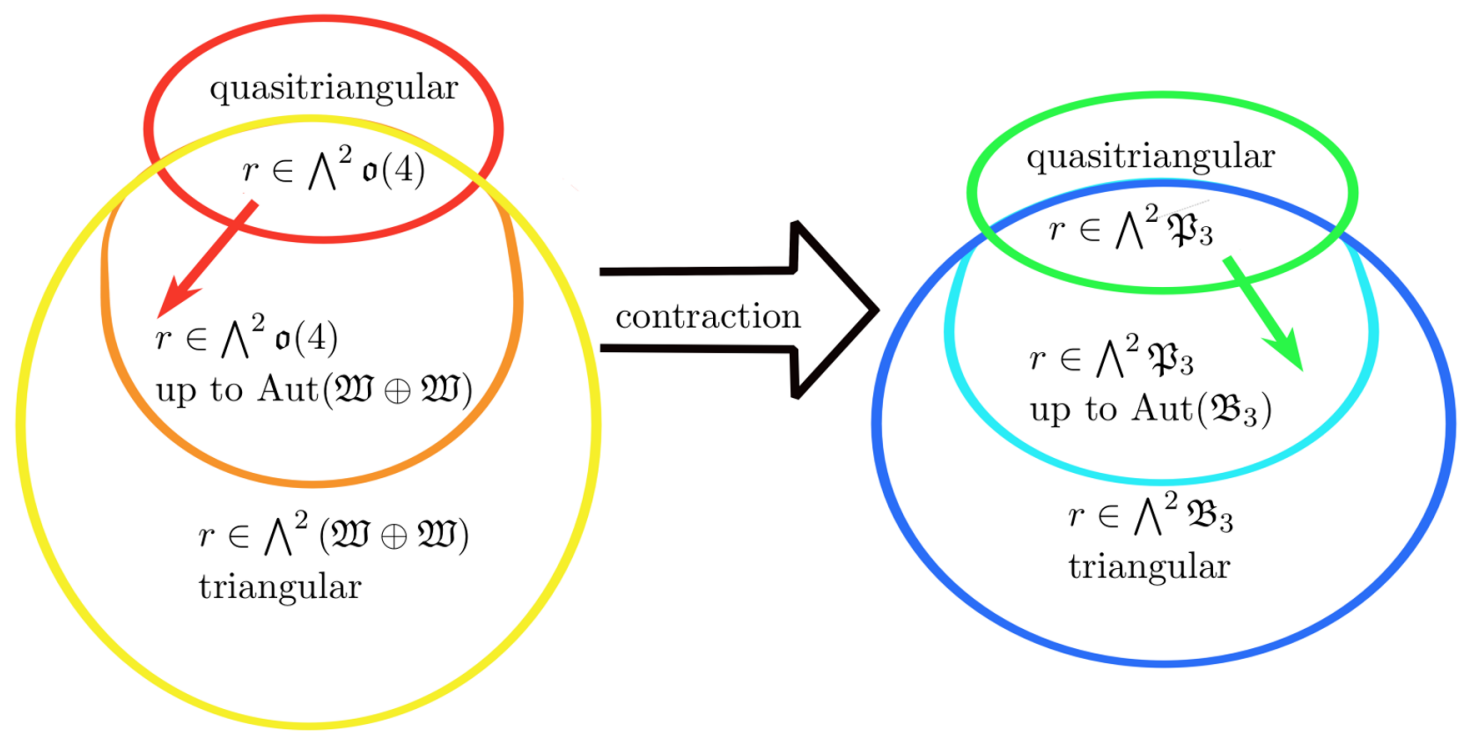

Figure 1. Schematic depiction of r-matrices in three dimensional asymptotic symmetry algebras. The red arrow represents the quotient $\operatorname{Aut}(\mathfrak{o}(4)) / \operatorname{Aut}(\mathfrak{W} \oplus \mathfrak{W})$ and the green arrow $\operatorname{Aut}\left(\mathfrak{P}_{3}\right) / \operatorname{Aut}\left(\mathfrak{B}_{3}\right)$. All the r-matrices inside the yellow and blue circle are triangular.

It is also not clear a priori if the contraction from (4.12)-(4.19) to this set is surjective and one has to take into account that the contraction limit can be performed along different axis as explained in the following. The (anti) de Sitter algebra (2.5)-(2.7) is isomorphic to

$$
\left[M_{A B}, M_{C D}\right]=\delta_{A C} M_{B D}-\delta_{B C} M_{A D}+\delta_{B D} M_{A C}-\delta_{A D} M_{B C},
$$

where the indices range from 1 to 4 and

$$
\begin{aligned}
M_{+-} & =M_{13}, & M_{+2} & =M_{12}+M_{32}, \quad M_{-2}=M_{12}-M_{32} \\
K_{ \pm} & =M_{14} \pm M_{34}, & K_{2} & =M_{24},
\end{aligned}
$$

i.e. the fourth axis is chosen for contraction. With the isomorphism

$$
\begin{aligned}
H & =-\frac{i}{2}\left(M_{12}+M_{34}\right), \quad \bar{H}=\frac{i}{2}\left(M_{12}-M_{34}\right), \\
E_{ \pm} & =-\frac{i}{2}\left(M_{23}+M_{14}\right) \mp \frac{1}{2}\left(M_{24}-M_{13}\right), \\
\bar{E}_{ \pm} & =\frac{i}{2}\left(M_{23}-M_{14}\right) \mp \frac{1}{2}\left(-M_{24}-M_{13}\right)
\end{aligned}
$$

one can express the r-matrices in terms of $M_{A B}$. Depending on which axis is chosen the result of the contraction differs, e.g. when choosing the second instead of the fourth axis

$$
K_{i}=M_{i 2}, \quad J_{i}=\epsilon_{i j k} M_{j k}
$$

one finds for

$$
r=\left(E_{+}+\bar{E}_{+}\right) \wedge(H+\bar{H})
$$


which is automorphic to $r_{I}$,

$$
r=\frac{i}{2}\left(i J_{3}+J_{4}\right) \wedge J_{1}
$$

where the contraction limit can be taken without rescaling. The $J_{i}$ satisfy

$$
\left[J_{1}, J_{3}\right]=J_{4}, \quad\left[J_{1}, J_{4}\right]=J_{3}, \quad\left[J_{3}, J_{4}\right]=J_{1}
$$

and can thus be related to the three dimensional Lorentz sector via

$$
J_{3}=-l_{0}, \quad J_{1}=\frac{l_{1}+l_{-1}}{2}, \quad J_{4}=\frac{l_{1}-l_{-1}}{2}
$$

leading to

$$
\hat{r}=l_{1} \wedge l_{-1}+l_{0} \wedge\left(l_{1}+l_{-1}\right) .
$$

Note that this r-matrix of the $\mathfrak{P}_{3}$ can not be obtained from a contraction of (4.12)-(4.19) which are associated with the fourth axis.

But even after taking into account the possibility to contract along different axis it turns out that there are triangular r-matrices in $\mathfrak{P}_{3}$, e.g.

$$
r=l_{0} \wedge T_{1}+\Theta_{1} T_{1} \wedge T_{0}+\Theta_{2} T_{1} \wedge T_{-1}
$$

that can not be obtained in its general from a contraction of a triangular r-matrix. This is important insofar it would enable a constructive method to obtain a twist for all deformations as we will see now.

Namely there is the possibility in performing the contraction limit on the level of the full twist. As an example let us consider the light-cone twist

$$
\mathcal{F}_{\mathrm{LC}}=e^{L_{0} \otimes \log \left(1+a L_{n}\right)} e^{\bar{L}_{0} \otimes \log \left(1-a \bar{L}_{n}\right)}
$$

corresponding also to (4.4). We express the $L_{m}, \bar{L}_{m}$ in terms of $l_{m}, T_{m}$

$$
\begin{aligned}
\mathcal{F}_{\mathrm{LC}}= & \exp \left(\frac{1}{2}\left(l_{0}+\frac{T_{0}}{\sqrt{-\Lambda}}\right) \otimes \log \left(1+\frac{a}{2}\left(l_{n}+\frac{T_{n}}{\sqrt{-\Lambda}}\right)\right)\right) \\
& \times \exp \left(\frac{1}{2}\left(l_{0}-\frac{T_{0}}{\sqrt{-\Lambda}}\right) \otimes \log \left(1-\frac{a}{2}\left(l_{n}-\frac{T_{n}}{\sqrt{-\Lambda}}\right)\right)\right) .
\end{aligned}
$$

In order to obtain a finite contraction limit we have to rescale $a \rightarrow a^{\prime}=a / \sqrt{-\Lambda}$. Expanding the first exponent of the twist in powers of $\sqrt{-\Lambda}$ and taking the limit then results in

$$
\begin{aligned}
& \frac{1}{2}\left(l_{0}+\frac{T_{0}}{\sqrt{\Lambda}}\right) \otimes \sum_{j=1} \frac{-\left(-a^{\prime}\right)^{j}}{j}\left(\sqrt{-\Lambda} l_{n}+T_{n}\right) \\
& \quad=\frac{1}{2} l_{0} \otimes \log \left(1+a^{\prime} T_{n}\right)+\frac{1}{2} T_{0} \otimes \sum_{j=1} \frac{-\left(-a^{\prime}\right)^{j}}{j} j l_{n} T_{n}^{j-1}+\mathcal{O}(\sqrt{-\Lambda}) \\
& \quad=\frac{1}{2} l_{0} \otimes \log \left(1+a^{\prime} T_{n}\right)+\frac{1}{2} T_{0} \otimes l_{n} a^{\prime}\left(1+a^{\prime} T_{n}\right)^{-1} .
\end{aligned}
$$


After repeating this procedure for the second exponent the final twist is given by

$$
\mathcal{F}_{\mathrm{LC}}=\exp \left(l_{0} \otimes \log \left(1+a^{\prime} T_{n}\right)+a^{\prime} T_{0} \otimes l_{n}\left(1+a^{\prime} T_{n}\right)\right) .
$$

It automotically satisfies the 2 -cocycle condition in $\mathfrak{B}_{3}$ since the twist (4.74) satisfies it in $\mathfrak{W} \oplus \mathfrak{W}$.

Similarly from the contraction of the abelian twist discussed in section 4.1.1 we obtain the abelian twist discussed in [28], where its physical interpretation is discussed and compared to the Jordanian twist deformation.

(Non-)uniqueness of the twist. From [28] we also know that the extended Jordanian twist of the form

$$
\mathcal{F}_{\mathrm{eJ}}=\exp \left(\frac{a^{\prime \prime}}{2} l_{n} \otimes T_{0}\right) \exp \left(-\frac{l_{0}}{n} \otimes \log \left(1+a^{\prime \prime} n T_{n}\right)\right)
$$

exists for the $\mathfrak{B}_{3}$ r-matrix $r=l_{0} \wedge T_{n}+l_{n} \wedge T_{0}$ which is the contraction limit of (4.3). Comparing the two twists reveals that they are related by a flip in first order and differ in higher orders. However, the inequivalence is only superficial as we have to take into account automorphisms on the universal envelope. We find there exist invertible elements $\omega \in U \mathfrak{B}_{3}[[1 / \kappa]]$ inducing the automorphisms

$$
f\left(l_{m}\right)=\omega^{-1} l_{m} \omega, \quad f\left(T_{m}\right)=\omega^{-1} T_{m} \omega
$$

by a similarity transformation. In general for every twist deformed Hopf algebra with $\mathcal{F}$ one can obtain a gauge equivalent twist via $\mathcal{F}_{\omega}=\omega^{-1} \otimes \omega^{-1} \mathcal{F} \Delta(\omega)$. The new twist then satisifies the 2-cocycle condition because

$$
\begin{aligned}
\mathcal{F}_{\omega 12}(\Delta \otimes 1)\left(\mathcal{F}_{\omega}\right) & \left.=\left(\omega^{-1} \otimes \omega^{-1}\right) F_{12}(\Delta(\omega) \otimes \omega) \Delta\left(\omega^{-1}\right) \otimes \omega^{-1}\right)(\Delta \otimes 1) \mathcal{F}(\Delta(\omega) \otimes \omega) \\
& =\left(\omega^{-1} \otimes \omega^{-1}\right) \mathcal{F}_{23}(1 \otimes \Delta) \mathcal{F}(\Delta(\omega) \otimes \omega) \\
& =\mathcal{F}_{\omega 23}(1 \otimes \Delta) \mathcal{F}_{\omega}
\end{aligned}
$$

and $f(x)=\omega x \omega^{-1}$ establishes the isomorphism between the twisted Hopf algebras

$$
\Delta_{\mathcal{F}} \circ f=(f \otimes f) \circ \Delta_{\mathcal{F}_{\omega}} .
$$

If the untwisted Hopf algbebra admits a $*$-structure the twist has to satisfy

$$
\mathcal{F}^{* \otimes *}=\mathcal{F}^{-1},
$$

i.e. be unitary in order to preserve the $*$-structure. On the invertible element $\omega$ this enforces the unitarity condition as well

$$
\begin{aligned}
& \mathcal{F}_{\omega}^{* \otimes *}=\Delta\left(\omega^{*}\right) \mathcal{F}^{-1}\left(\omega^{*-1} \otimes \omega^{*-1}\right) \stackrel{!}{=} \mathcal{F}_{\omega}^{-1}=\Delta\left(\omega^{-1}\right) \mathcal{F}^{-1}(\omega \otimes \omega) \\
& \Leftrightarrow \omega^{*}=\omega^{-1} .
\end{aligned}
$$

In our particular example we find that in first order of $1 / \kappa$ the isomorphism induced from the element

$$
\omega=e^{-\frac{a^{\prime}}{4}\left(l_{n} T_{0}+T_{0} l_{n}\right)}
$$

relates the extended Jordanian and the contraction limit of the light-cone twist for $a^{\prime \prime}=$ $-a^{\prime}$. It is easy to see that it is hermitian with respect to the reality condition $l_{m}^{*}=$ $-l_{m}, T_{m}^{*}=-T_{m}$ and $a^{\prime} \in i \mathbb{R}$. 


\subsection{Deformations of the surface charge algebra}

As we noted in section 2.3 the algebra of the surface charges in an asymptotically AdS spacetime differs from the previously examined $\mathfrak{W} \oplus \mathfrak{W}$ by a central extension. Therefore we want to investigate whether this has any impact on the possible deformations.

First let us note without proof that the first cohomology group $H^{1}(\mathfrak{V i r} \oplus \mathfrak{V i r}, \wedge \mathfrak{V i r} \oplus$ $\mathfrak{W})$ vanishes and there are again no ad-invariant elements in the exterior product. As a consequence all LBA are coboundary and we will now investigate how the classification of the r-matrices is affected. Note that there is no automorphism on $\mathfrak{V i r} \oplus \mathfrak{V i r}$ that mixes elements of $\mathfrak{W}$ with central elements so it is enough to consider (4.12)-(4.19). More exactly, the formulas (4.10) are still valid if we assume $\phi\left(c_{L}\right)=c_{L}$. By a straightforward computation one finds that all of these r-matrices are still triangular except for those containing $a_{1}$ if we choose a different embedding. In particular for $a_{1}$ with $L_{ \pm 1} \rightarrow L_{ \pm n}$ we have

$$
\left[\left[a_{1}, a_{1}\right]\right]=\frac{\left(n^{3}-n\right)}{6}\left(L_{n} \wedge c_{L} \wedge L_{-n}+\wedge c_{L} \wedge L_{0}+L_{-n} \wedge c_{L} \wedge L_{0}\right),
$$

so it no longer defines a LBA.

Furthermore it is easy to see that all $r \in c_{L} \wedge(\mathfrak{W} \oplus \mathfrak{W})$ are valid r-matrices. By calculating the Schouten brackets of combinations with r-matrices from (4.12)-(4.19) only r-matrices of the form

$$
\begin{aligned}
& r=c_{L} \wedge L_{p}+L_{p} \wedge\left(\sum_{q} \bar{L}_{q}\right), \\
& r=c_{L} \wedge L_{p}+\bar{r}, \\
& r=c_{L} \wedge L_{p}+\alpha L_{p} \wedge L_{0}
\end{aligned}
$$

are possible, where $\bar{r}$ is a triangular r-matrix generated by $\bar{L}_{q}$.

The central extension thus can impact the LBAs. For example with $r=c_{L} \wedge L_{p}$ we obtain

$$
\delta_{r}\left(L_{m}\right)=c_{L} \wedge(m-p) L_{m+p}, \quad \delta_{r}\left(\bar{L}_{m}\right)=0 .
$$

But also Lie bialgebras from r-matrices that contain no central element can contribute extra terms, e.g. $r=\chi L_{0} \wedge L_{n}$ yields

$$
\delta_{r}\left(L_{-n}\right)=-n \chi L_{-n} \wedge L_{n}-\chi \frac{\left(n^{3}-n\right)}{12} L_{0} \wedge c_{L} .
$$

For all r-matrices from (4.86)-(4.88) we can write down the twist. This is easy to see in the first two cases as everything is abelian. In the last case note that we can obtain the twist from the Jordanian twist by simply redefining $L_{0} \rightarrow L_{0}-\alpha c_{L}$ which leaves $\left[L_{0}, L_{p}\right]$ invariant.

From the twists we can directly compute the coalgebra structures. In general these will contain infinite expressions which, as we will explore in the next section, could be remedied by considering one-sided algebras. Even though this works in the case of the 
Witt algebra, with a central extension the one-sided algebra is pointless to consider as the central elements do not appear in the algebra sector. Thus out of (4.86)-(4.88) only $r=c_{L} \wedge\left(\chi L_{0}+\bar{\chi} \bar{L}_{0}\right)$ leads to a finite coalgebra sector. In particular,

$$
\Delta\left(L_{m}\right)=L_{m} \otimes 1+\exp \left(-m \chi c_{L}\right) \otimes L_{m}
$$

\section{One-sided Witt algebra and specialization}

So far the Hopf algebras we considered were defined with the $h$-adic topology and thus allowed for infinite power series in the formal parameter $1 / \kappa$. While this is mathematically consistent it is ultimately problematic when interpreting the formalism in a physical context where $1 / \kappa$ is to be identified with an energy scale of the order of the Planck mass. The problem of finding a Hopf algebra (the so-called q-analog) with the same (co)algebra structure where the formal parameter can be specialized to a complex (or real) parameter is known as specialization [48, 52]. Most importantly, all the structures in the q-analog need to be finite power series in the generators.

Let us study the specialization on the examples of the abelian and the Jordanian twist respectively. For the abelian twist the formulas (4.29)-(4.31) show that only a finite number of generators appear in the coproducts but there are infinite power series in $a \equiv \frac{i m}{\kappa n^{2}}$. Thus the full $\mathfrak{W} \oplus \mathfrak{W}$ can be turned into the q-analog by adding the elements

$$
e^{a L_{0}} \equiv K, \quad e^{-a L_{0}} \equiv K^{-1}, \quad e^{a \bar{L}_{0}} \equiv \bar{K}, \quad e^{-a \bar{L}_{0}} \equiv \bar{K}^{-1},
$$

to the algebra. Furthermore, define $q=e^{a}$ and the extra commutation relations become

$$
\begin{aligned}
e^{a L_{0}} L_{m} e^{-a L_{0}} & =\sum_{j=0}^{\infty} \frac{a^{j}}{j !}\left[L_{0},\left[\ldots,\left[L_{0}, L_{m}\right] \ldots\right]=e^{-a m} L_{m}=q^{-m} L_{m}\right. \\
\Rightarrow\left[K, L_{m}\right] & =q^{-m} L_{m} e^{a L_{0}}-L_{m} e^{a L_{0}}=\left(q^{-m}-1\right) L_{m} K
\end{aligned}
$$

and similarly

$$
\begin{array}{rlrl}
{\left[K^{-1}, L_{m}\right]} & =\left(q^{m}-1\right) L_{m} K^{-1}, & {\left[K, \bar{L}_{m}\right]} & =0, \\
{\left[K^{-1}, \bar{L}_{m}\right]} & =0, & {\left[\bar{K}, \bar{L}_{m}\right]} & =\left(q^{-m}-1\right) \bar{L}_{m} \bar{K} \\
{\left[\bar{K}^{-1}, L_{m}\right]} & =0, & {\left[\bar{K}^{-1}, \bar{L}_{m}\right]} & =\left(q^{m}-1\right) \bar{L}_{m} \bar{K}^{-1} \\
{\left[K, K^{-1}\right]} & =[K, \bar{K}]=\left[K, \bar{K}^{-1}\right]=0 . &
\end{array}
$$

It is also easy to compute

$$
\begin{aligned}
\Delta_{\mathcal{F}_{A}}(K) & =K \otimes K, & \Delta_{\mathcal{F}_{A}}\left(K^{-1}\right) & =K^{-1} \otimes K^{-1}, \\
S_{\mathcal{F}_{A}}(K) & =-K, & S_{\mathcal{F}_{A}}\left(K^{-1}\right) & =-K^{-1}
\end{aligned}
$$

and reexpressing (4.29)-(4.31) gives

$$
\begin{aligned}
& \Delta_{\mathcal{F}_{A}}\left(L_{m}\right)=K^{m} \bar{K}^{m} \otimes L_{m}+L_{m} \otimes K^{-m} \bar{K}^{m} \\
& \Delta_{\mathcal{F}_{A}}\left(\bar{L}_{m}\right)=K^{-m} \bar{K}^{-m} \otimes \bar{L}_{m}+\bar{L}_{m} \otimes K^{m} \bar{K}^{-m} .
\end{aligned}
$$


Endowed with this algebra and coalgebra structures the set of polynomials in the generators $\left\{L_{m}, \bar{L}_{m}, K, K^{-1}, \bar{K}, \bar{K}^{-1}\right\}$ does indeed form a q-analog of the twisted Hopf algebra and it can be defined for any $q \in \mathbb{C}$. In particular the classical limit $\kappa \rightarrow \infty \leftrightarrow q \rightarrow 1$ gives simply the Lie algebra $\mathfrak{W} \oplus \mathfrak{W}$ but extended by the central elements $K, \bar{K}$.

In the case of the Jordanian twist the situation is different. We discovered in (4.41) that for $L_{m}, m \in \mathbb{Z}$ the coproduct contains infinitely many different generators and thus it would be impossible to define a q-analog. However, by restricting to two copies of the one-sided Witt algebra $\mathfrak{W}_{-}$containing $L_{m}, m \leq 1$ it was shown that all coproducts contain finitely many terms. Similarly one could use the embedding corresponding to $n=-1$ and restrict to $\mathfrak{W}_{+}$containing $L_{m}, m \geq-1$. In order to express all algebra and coalgebra relations involving only finite powers of $1 / \kappa$ the elements $\Pi_{+}$, defined in (4.38) and its inverse $\Pi_{+}^{-1}$ are used. The additional commutation relations then read

$$
\begin{aligned}
{\left[\Pi_{+}, L_{m}\right] } & =\tilde{a}(1-m) L_{m+1}, \\
{\left[\Pi_{+}^{-1}, L_{m}\right] } & =\sum_{j=0}^{\infty} \frac{(-1)^{j}}{j !} \tilde{a}^{j}\left[\left(L_{1}-\bar{L}_{1}\right)^{j}, L_{m}\right] \\
& =\sum_{j=0}^{\infty} \sum_{k=1}^{\min \{1-m, j\}} \frac{(-1)^{j}}{j !}\left(\begin{array}{l}
j \\
k
\end{array}\right) L_{m+k} \tilde{a}^{j}\left(L_{1}-\bar{L}_{1}\right)^{j-k}\left(\prod_{r=0}^{k-1}(1-m-r)\right) \\
& =\sum_{k=1}^{1-m} L_{m+k} \frac{d^{k} e^{-\sigma_{1}}}{d L_{1}^{k}}\left(\prod_{r=0}^{k-1}(1-m-r)\right) \\
& =\sum_{k=1}^{1-m} \frac{(-1)^{\underline{k}}}{k !} \tilde{a}^{k} L_{m+k} \Pi_{+}^{k-1}\left(\prod_{r=0}^{k-1}(1-m-r)\right)
\end{aligned}
$$

and similarly

$$
\begin{aligned}
{\left[\Pi_{+}, \bar{L}_{m}\right] } & =-\tilde{a}(1-m) \bar{L}_{m+1}, \\
{\left[\Pi_{+}^{-1}, \bar{L}_{m}\right] } & =\sum_{k=1}^{1-m} \frac{(-1)^{\underline{k}}}{k !}(-\tilde{a})^{k} \bar{L}_{m+k} \Pi_{+}^{k-1}\left(\prod_{r=0}^{k-1}(1-m-r)\right) .
\end{aligned}
$$

From (4.50) one has in particular

$$
\Delta_{\mathcal{F}_{J}}\left(L_{1}\right)=L_{1} \otimes \Pi_{+}+\otimes L_{1}, \quad \Delta_{\mathcal{F}_{J}}\left(L_{1}\right)=\bar{L}_{1} \otimes \Pi_{+}+\otimes \bar{L}_{1},
$$

leading to

$$
\begin{aligned}
\Delta_{\mathcal{F}_{J}}\left(\Pi_{+}\right) & =\Pi_{+} \otimes \Pi_{+}, & \Delta_{\mathcal{F}_{J}}\left(\Pi_{+}^{-1}\right) & =\Pi_{+}^{-1} \otimes \Pi_{+}^{-1}, \\
S_{\mathcal{F}_{J}}\left(\Pi_{+}\right) & =-\Pi_{+}, & S_{\mathcal{F}_{J}}\left(\Pi_{+}^{-1}\right) & =-\Pi_{+}^{-1} .
\end{aligned}
$$

All these formulas are well defined for $\tilde{a} \in \mathbb{C}$ and for $\kappa \rightarrow \infty$ the elements $\Pi_{+}, \Pi_{+}^{-1}$ become central. Thus, similar to the abelian twist, the classical limit is the centrally extended Lie algebra $\mathfrak{W}_{+} \oplus \mathfrak{W}_{+}$.

Additionally, if we consider the centrally extended algebra of surface charges, the rmatrix $r=c_{L} \wedge\left(\chi L_{0}+\bar{\chi} \bar{L}_{0}\right)$ with the twist $\mathfrak{F}=\exp \left(c_{L} \otimes\left(\chi L_{0}+\bar{\chi} \bar{L}_{0}\right)\right)$ induces a Hopf algebra 
which admits a specialization by simply adding the elements $\Pi=\exp \left(\chi c_{L}\right), \bar{\Pi}=\exp \left(\bar{\chi} c_{L}\right)$. These elements are thus just redefinitions of the Brown-Henneaux central charge.

It turns out that all twist deformations except for the abelian twist do not have a q-analog on the full Witt algebras. But those (and only those) which do not contain both $L_{1}$ and $L_{-1}$ or $\bar{L}_{1}$ and $\bar{L}_{-1}$ simultaneously can be shown to permit specialization on the one-sided Witt algebras in a similar way as for the Jordanian twist. Therefore we will investigate what physical implications the restriction of the generators has.

To this end we consider an asymptotically flat spacetime in four dimensions, described by $\mathfrak{B}_{4}$ (see also [28]). Recall that the superrotation Killing vectors are parametrized by functions $R^{A}$ on the sphere. For $m \geq-1$ these functions do not contain negative powers of $z, \bar{z}$ and are thus holomorphic on the whole sphere except for $z=\infty$. Note that only the ordinary rotations with $m=0,1,2$ are globally defined as the vectorfields $R_{m}^{z} \equiv z^{m} \partial_{z}, m<$ 0 and, after redefining $\omega=z^{-1}, R_{m}^{z}=\omega^{2-m} \partial_{\omega}, m>2$ have a singularity at the origin [46].

In contrast, consider the following construction due to Penrose where Minkowski space is cut along the light-cone $u=0[53,54]$. Then, after performing a diffeomeorphism on the $u>0$ patch, it is glued together such that the metric is continuous at $u=0$. That procedure introduces singularities and was later linked to cosmic strings [55]. A cosmic string is a topological defect with dimension one and is conjectured to exist if in the early universe the topology was not simply connected. The geometry containing a cosmic string is not exactly asymptotically flat because of the singularities but it satisfies a weaker requirement and is said to be asymptotically locally flat. A snapping string with ends at $z=0, \infty$ that starts to snap from $u=0$ is indeed described by Penrose' construction and furthermore one can show that certain superrotations of flat space yield cosmic strings. In other words a superrotation that is only meromorphic, i.e. isolated singularities are allowed, maps a flat geometry to a flat geometry except at the singularities [54].

If the results we obtained in three dimensions carry over qualitatively to the four dimensional case, i.e. that the consistent specialization of particular twist deformations requires the restriction to the one-sided $\mathfrak{B}_{4+}$, then we could conclude that the remaining superrotations do not allow for the formation or decay of cosmic strings. Thus phenomenological evidence for the existence of cosmic strings, e.g. from observing gravitational wave signatures of their decay, could be used to constrain theories of quantum groups and noncommutative geometry.

\section{Conclusion}

It was shown in this work that all Lie bialgebra structures on the symmetry algebra of asymptotically (A)dS spacetime in 3 dimensions are coboundary and triangular and can thus be quantized with the help of the Drinfeld twist technique. Physically viable rmatrices, that is those which are compatible with singling out an embedding representing a vacuum choice, are all classified. Also the triangularity condition constrains the possible Lie bialgebras and in particular some of the structures that are defined on the 3 -dimensional Poincaré algebra related to $\kappa$-Poincaré quantum groups are eliminated due to this. With the help of the quantization of the Lie bialgebra structures on (real forms of) $\mathfrak{o}(4, \mathbb{C})$ there 
is a constructive way to obtain the associated Hopf algebras in all orders of the deformation parameter also for the revised classes of r-matrices in the infinite-dimensional $\mathfrak{W} \oplus \mathfrak{W}$ and its $\mathrm{BMS}_{3}$ contraction limit, cf. a schematic overview presented in figure 1. When performing this twist procedure it becomes apparent that the specialization of the formal deformation parameter to real values can not be done for all Hopf algebras. Rather, in these cases, this is only possible when a subalgebra of the asymptotic symmetry algebra is considered. We propose that this would have testable consequences when transferred to a realistic setting, namely the existence of cosmic strings would be inconsistent with the quantum group deformations. Further phenomenological consequences were already studied for the flat case in [28] and we make contact with this work by performing a contraction limit.

There is a number of problems that we hope to be able to address in a future. First of all it would be of great interest to directly derive the deformation of 3 dimensional BMS algebra by using the non-perturbative methods similar to those that made it possible to derive the deformation of quantum AdS algebra of charges in [30]. Given that by $\mathrm{AdS}_{3} / \mathrm{CFT}_{2}$ asymptotic symmetries of gravity with negative cosmological constant should correspond to the symmetries of the conformal field theory on the asymptotic boundary of spacetime it is natural to investigate CFTs with deformed conformal symmetries, to understand, among others, what would be the origin of the deformation, corresponding to the non-perturbative quantum gravity effect leading to the deformation of symmetries in the bulk.

In this paper we considered only deformations of the $\Lambda$-BMS 3 algebra (2.3)-(2.4). Recently, various generalizations of this algebra were proposed, which result from boundary conditions different from the Brown-Henneaux ones [7] adopted here. In the paper [57] the authors consider chiral boundary conditions and the resulting algebra of charges differs from (2.3)-(2.4). It would be of interest to look for possible deformations of this algebra, however since the translational sector of it differs from the one we consider, to do so one has to adopt a new class of twists. Another generalization of the $\Lambda$-BMS 3 algebra considered here was proposed recently in,Fuentealba:2020zkf where the conformal extension of BMS algebra were considered. Since $\mathfrak{o}(3,1)$ is a subalgebra of the three dimensional conformal algebra $\mathfrak{o}(3,2)$, to deform the latter one can readily use the twists constructed here. In the case of the conformal $\mathrm{BMS}_{3}$ there are also other twist deformations, which it would be of interest to investigate in some details One should notice however that in contrast to $\mathfrak{o}(4, \mathbb{C})$ case not all quantum deformations of $\mathfrak{o}(5, \mathbb{C})$ algebra are yet known. Other proposals leading to different algebras that can be, in principle, deformed using the method presented in this paper have been reported in the papers,Afshar:2016wfy,Grumiller:2019fmp,Batlle:2020hia,Adami:2020ugu.

In this paper we considered only the 3-dimensional model of non-zero cosmological constant $\Lambda$-BMS algebra. The reason why we choose to investigate here the simpler 3dimensional $\Lambda$-BMS algebra is that in 4 dimensions the non-vanishing cosmological constant extension of the BMS algebra has the structure of a Lie algebroid, with structure functions instead of structure constants [41, 43, 44, 63]. Despite some attempts to generalize the notions of quantum groups/Lie bialgebras to bialgebroids/Hopf algebroids [64-66] there is no established concept for deformations of Lie algebroids. 


\section{Acknowledgments}

This work is supported by funds provided by the Polish National Science Center (NCN), project UMO-2017/27/B/ST2/01902 and for JKG, and JU also by the project number 2019/33/B/ST2/00050.

\section{A Proof of the cohomology theorems}

\section{A.1 Proof of theorem 1}

We start by noting that the 1 -cocycles $\delta$ can be separated by their degree $d \in \mathbb{Z}$. This degree is derived from the grading of $\mathfrak{W}$, i.e.

$$
\delta\left(L_{m}\right)=L_{i} \wedge L_{j}
$$

has degree $d=i+j-m$. The separation by degree follows from the fact that a cocycle which, applied to elements of $\mathfrak{W}$, results in terms with different degree can be split into cocycles of homogeneous degree which have to fulfill the cocycle condition (3.10) independently. Let us first consider cocycles of degree $d \neq 0$. We will show that all such cocycles $\delta$ are cohomolog to 0 , i.e. that $\delta^{\prime}\left(L_{m}\right)=\delta\left(L_{m}\right)-\left(\partial_{0} r\right)\left(L_{m}\right)=0$ for all $m \in \mathbb{Z}$. Let $\delta$ be a cocycle of homogenous degree $d$ such that

$$
\delta\left(L_{m}\right)=\sum_{i_{m}, j_{m} \in I_{m}} \alpha_{i_{m} j_{m}}^{m} L_{i_{m}} \wedge L_{j_{m}}
$$

where $\alpha_{i_{m} j_{m}}^{m} \in \mathbb{R} ; i_{m}, j_{m} \in \mathbb{Z}$ and $I_{m}$ are finite subsets of $\mathbb{Z}$. Choose a 0 -cochain

$$
r=-\sum_{i_{0}, j_{0} \in I_{0}} \frac{\alpha_{i_{0} j_{0}}^{0}}{i_{0}+j_{0}} L_{i_{0}} \wedge L_{j_{0}} .
$$

Then we have

$$
\begin{aligned}
\delta^{\prime}\left(L_{0}\right) & =\sum_{i_{0}, j_{0} \in I_{0}} \alpha_{i_{0}, j_{0}}^{0} L_{i_{0}} \wedge L_{j_{0}}-\left[L_{0} \otimes 1+1 \otimes L_{0}, r\right] \\
& =\sum_{i_{0}, j_{0} \in I_{0}} \alpha_{i_{0}, j_{0}}^{0} L_{i_{0}} \wedge L_{j_{0}}-\sum_{i_{0}, j_{0} \in I_{0}} \frac{\alpha_{i_{0} j_{0}}^{0}}{i_{0}+j_{0}}\left(i_{0}+j_{0}\right) L_{i_{0}} \wedge L_{j_{0}}=0 .
\end{aligned}
$$

From the cocycle condition

$$
\delta^{\prime}\left(\left[L_{0}, L_{m}\right]\right)=\left[L_{0} \otimes 1+1 \otimes L_{0}, \delta^{\prime}\left(L_{m}\right)\right]-\left[L_{m} \otimes 1+1 \otimes L_{m}, \delta^{\prime}\left(L_{0}\right)\right],
$$

for $m \neq 0$, we infer

$$
\begin{aligned}
m \delta\left(L_{m}\right) & =\sum_{i_{m}, j_{m} \in I_{m}}\left(i_{m}+j_{m}\right) \alpha_{i_{m} j_{m}}^{m} L_{i_{m}} \wedge L_{j_{m}} \\
& =(d+m) \delta^{\prime}\left(L_{m}\right) \\
\Rightarrow \delta^{\prime}\left(L_{m}\right) & =0
\end{aligned}
$$


which concludes the proof for cocycles of degree $d \neq 0$.

Next, let us consider cocycles of degree $d=0$ which can be written in the form

$$
\delta\left(L_{m}\right)=\sum_{i_{m} \in I} \gamma_{i_{m}}^{m} L_{m-i_{m}} \wedge L_{i_{m}}
$$

Note that without loss of generality we can restrict the indices $i_{m}$ to be smaller than $m / 2$ since otherwise, i.e. if there is an index $i_{m}>m / 2$, we simply substitute $i_{m}^{\prime}=m-i_{m}$ and $\gamma_{i_{m}^{\prime}}^{\prime m}=\gamma_{i_{m}}^{m}-\gamma_{i m-m}^{m}$ to describe the same cocycle. We will make repeated use of this in the rest of the proof.

The conditions

$$
\begin{aligned}
\delta\left(\left[L_{0}, L_{m}\right]\right) & =\left[L_{0} \otimes 1+1 \otimes L_{0}, \delta\left(L_{m}\right)\right]-\left[L_{m} \otimes 1+1 \otimes L_{m}, \delta\left(L_{0}\right)\right] \\
& =(-m) \delta\left(L_{m}\right)-\left[L_{m} \otimes 1+1 \otimes L_{m}, \delta\left(L_{0}\right)\right] \\
\Leftrightarrow 0 & =\left[L_{m} \otimes 1+1 \otimes L_{m}, \delta\left(L_{0}\right)\right]
\end{aligned}
$$

implie that all degree 0 cocycles vanish on $L_{0}$ because it has to hold for all $m$ and there is no ad-invariant element in $\bigwedge^{2}(\mathfrak{W} \oplus \mathfrak{W})$.

As a next step we show that all cocycles are cohomolog to 0 on $L_{ \pm 1}$. Let us assume without loss of generality that the indices of

$$
\delta\left(L_{1}\right)=\sum_{i_{1} \in I_{1}} \gamma_{i_{1}}^{1} L_{1-i_{1}} \wedge L_{i_{1}}
$$

are given by $i_{1} \in I_{1}=\left\{-p_{1},-p_{2}, \ldots,-p_{n} \mid p_{1}>p_{2}>\ldots>p_{n}>1, n \in \mathbb{N}\right\}$. From the cocycle condition we get

$$
\begin{aligned}
\delta\left(\left[L_{1}, L_{-1}\right]\right)= & {\left[L_{1} \otimes 1+1 \otimes L_{1}, \delta\left(L_{-1}\right)\right]-\left[L_{-1} \otimes 1+1 \otimes L_{-1}, \delta\left(L_{1}\right)\right] } \\
\Leftrightarrow 0= & \sum_{i_{-1} \in I_{-1}}\left(\gamma_{i_{-1}}^{-1}\left(2+i_{-1}\right) L_{-i_{-1}} \wedge L_{i_{-1}}+\gamma_{i_{-1}}^{-1}\left(i_{-1}-1\right) L_{1+i_{-1}} \wedge L_{-1-i_{-1}}\right) \\
& +\sum_{j=1}^{n}\left(\gamma_{-p_{j}}^{1}\left(2+p_{j}\right) L_{p_{j}} \wedge L_{-p_{j}}+\gamma_{-p_{j}}^{1}\left(p_{j}-1\right) L_{-1-p_{j}} \wedge L_{1+p_{j}}\right) .
\end{aligned}
$$

Lets focus on the first term in the second line of (A.13) with $p_{1}$; it can only be cancelled by any of the other $p_{j}$ terms if $p_{2}=p_{1}-1$ which we discuss below. In the case $p_{2} \neq p_{1}-1$ there are two terms that can contribute, one from the first and the second summand in the first line in (A.13) which we will call type $I$ and type $I I$ terms respectively. ${ }^{6}$ The type $I I$ term would correspond to $i_{-1}=-1-p$. If it existed with non-zero $\gamma_{-1-p}^{-1}$ it would imply the existence of a type $I$ term of the form $\gamma_{-1-p}^{-1}\left(1-p_{1}\right) L_{1+p_{1}} \wedge L_{-1-p_{1}}$ which in turn can only be cancelled by a type $I I$ term with $i_{-1}=-2-p_{1}$. Since also none of the prefactors $\left(2+i_{-1}\right)$ and $\left(i_{-1}-1\right)$ vanishes if $p_{1} \neq 1$ this would go on forever so that we need infinitely many terms in $\delta\left(L_{-1}\right)$ which is not possible. Thus $\gamma_{-1-p}^{-1}=0$ and we need a type $I$ term with $i_{-1}=-p_{1}$

$$
\gamma_{1-p_{1}}^{-1}\left(2+p_{1}\right) L_{p_{1}} \wedge L_{-p_{1}}
$$

\footnotetext{
${ }^{6}$ Here and in the following we use the index restriction. Otherwise also e.g. a type $I$ term with $i_{-1}=p_{j}$ could be used.
} 
which implies a type $I I$ term with the same $i_{-1}$

$$
\gamma_{-p_{1}}^{-1}\left(-1-p_{1}\right) L_{1-p_{1}} \wedge L_{p_{1}-1}
$$

This term can be cancelled only by a type $I$ term with $i_{-1}=1-p_{1}$

$$
\gamma_{1-p_{1}}^{-1}\left(3-p_{1}\right) L_{1-p_{1}} \wedge L_{p_{1}-1}
$$

and the corresponding type $I I$ term

$$
\gamma_{1-p_{1}}^{-1} p_{1} L_{2-p_{1}} \wedge L_{p_{1}}
$$

requires again a type $I$ term with $i_{-1}=-p_{1}$

$$
\gamma_{-p_{1}}^{-1}\left(2-p_{1}\right) L_{2-p_{1}} \wedge L_{p_{1}}
$$

ending the sequence. The cancellation of (A.17) with (A.18) implies the following ratio of the coefficients

$$
\frac{\gamma_{-p_{1}}^{-1}}{\gamma_{1-p_{1}}^{-1}}=\frac{2-p_{1}}{p_{1}}
$$

and when considering the 0-cochain

$$
r=\gamma_{s} L_{-s} \wedge L_{s}
$$

with $s=1-p_{1}$, implying

$$
\left(\partial_{0} r\right)\left(L_{-1}\right) \equiv \delta_{r}\left(L_{-1}\right)=\gamma_{1-p_{1}}\left(\left(-p_{1}\right) L_{-2+p_{1}} \wedge L_{1-p_{1}}+\left(-2+p_{1}\right) L_{p_{1}-1} \wedge L_{-p_{1}},\right.
$$

we find the same ratio between the two summands. Thus setting $\gamma_{1-p_{1}}\left(p_{1}\right)=\gamma_{1-p_{1}}^{-1}$ in the cocycle

$$
\delta^{\prime}=\delta+\delta_{r}
$$

both coefficients $\gamma_{-p_{1}}^{-1}, \gamma_{1-p_{1}}^{\prime-1}$ vanish and therefore also $\gamma_{p_{1}}^{\prime 1}$ has to be zero.

Next, we have to consider the case $p_{2}=p_{1}-1$. In (A.13) the term

$$
\gamma_{-p_{1}}^{1}\left(p_{1}-1\right) L_{1+p_{1}} \wedge L_{-1-p_{1}}
$$

can be cancelled by a type $I$ term with $i_{-1}=1-p_{1}$ or a type $I I$ term with $i_{-1}=-2-p_{1}$. If the second term does not vanish it implies the existence of a type $I$ term with the same $i_{-1}$ which can only be eliminated by a type $I I$ term with $i_{-1}=-3-p_{1}$ and so on, so that infinitely many terms are necessary, ruling out this option. Using the same cochain as above in (A.20) with the same choice for $s$ and $\gamma_{s}$ we can eliminate the coefficient $\gamma_{1-p_{1}}^{\prime-1}$ and thus the possibility to cancel (A.23) with a type $I$ term is not possible which means that $\gamma^{\prime 1}{ }_{-1}$ has to vanish.

For the rest of the $p_{j}, j>1$ we can iteratively use the same argumentation. In particular the arguments with the infinite number of terms in $\delta\left(L_{-1}\right)$ can be extended to the 
higher $j$ as the sequence would stop at the $i_{-1}=-p_{j-1}$ terms which already has to vanish. Furthermore, one has to add coboundaries from the cochains

$$
r_{j}=\gamma_{j} L_{-\left(1-p_{j}\right)} \wedge L_{1-p_{j}}, \quad j>1
$$

with suitable coefficients $\gamma_{j}\left(p_{j}\right)=\gamma_{1-p_{j}}^{\prime \ldots .}$ where we define

$$
\delta^{\prime \prime}=\delta^{\prime}+\delta_{r_{2}}, \ldots
$$

iteratively so that the required terms in $\delta^{\prime \cdots \prime}\left(L_{-1}\right)$ are eliminated.

Finally, let us explicitely consider the case $p_{1}=1$ that was excluded in the argumentation above. In that case

$$
\delta\left(L_{1}\right)=\gamma_{-1}^{1} L_{0} \wedge L_{1}
$$

and from the cocycle condition we infer that

$$
\delta\left(L_{-1}\right)=\gamma_{-1}^{1} L_{-2} \wedge L_{1}
$$

On $L_{ \pm 1} \delta$ then coincides with $\delta_{r}$, where $r=\gamma_{-1}^{1} / 2 L_{1} \wedge L_{-1}$ and thus $\delta^{\prime}=\delta-\delta_{r}$ is zero on these elements. This concludes the proof that $\delta$ is cohomolog to 0 on $L_{1}$.

In the next step it will be shown that $\delta\left(L_{1}\right)=0$ implies that $\delta\left(L_{m}\right)=0$ for $m>1$. Starting from

$$
\delta\left(L_{2}\right)=\sum_{i_{2} \in I_{2}} \gamma_{i_{2}}^{2} L_{2-i_{2}} \wedge L_{i_{2}}
$$

one explicitely obtains by using (3.10) with $m=1, n=2, m=1, n=3$ and $m=1, n=4$

$$
\begin{aligned}
\delta\left(L_{3}\right)= & -\sum_{i_{2} \in I_{2}} \gamma_{i_{2}}^{2}\left(\left(i_{2}-1\right) L_{3-i_{2}} \wedge L_{i_{2}}-\left(1-i_{2}\right) L_{2-i_{2}} \wedge L_{i_{2}+1}\right) \\
\delta\left(L_{4}\right)= & \sum_{i_{2} \in I_{2}} \frac{\gamma_{i_{2}}^{2}}{2}\left(i_{2}-1\right)\left(\left(i_{2}-2\right) L_{4-i_{2}} \wedge L_{i_{2}}+2\left(1-i_{2}\right) L_{3-i_{2}} \wedge L_{i_{2}+1}-i_{2} L_{2-i_{2}} \wedge L_{i_{2}+2}\right) \\
\delta\left(L_{5}\right)= & -\sum_{i_{2} \in I_{2}} \frac{\gamma_{i_{2}}^{2}}{6}\left(i_{2}-1\right)\left(\left(i_{2}-2\right)\left(i_{2}-3\right) L_{5-i_{2}} \wedge L_{i_{2}}+3\left(1-i_{2}\right)\left(i_{2}-2\right) L_{4-i_{2}} \wedge L_{i_{2}+1}\right. \\
& \left.+3\left(1-i_{2}\right) i_{2} L_{3-i_{2}} \wedge L_{i_{2}+2}+i_{2}\left(1+i_{2}\right) L_{2-i_{2}} \wedge L_{i_{2}+3}\right) .
\end{aligned}
$$

Using the same argumentation as above we can restrict $i_{2}$ to be bigger than 1 and we 
consider the largest index $i_{2}^{\prime}$. Then, (3.10) with $m=2, n=3$ yields

$$
\begin{aligned}
0= & \sum_{i_{2} \in I_{2}}\left(L_{5-i_{2}} \wedge L_{i_{2}} \gamma_{i_{2}}^{2}\left(\frac{1}{6}\left(i_{2}-1\right)\left(i_{2}-2\right)\left(i_{2}-3\right)-\left(i_{2}-1\right)^{2}-\left(i_{2}^{\prime}+1\right)\right)\right. \\
& +L_{4-i_{2}} \wedge L_{i_{2}+1} \gamma_{i_{2}}^{2}\left(\frac{1}{2}\left(i_{2}-1\right)^{2}\left(2-i_{2}\right)+\left(i_{2}-1\right) i_{2}\right) \\
& +L_{3-i_{2}} \wedge L_{i_{2}+2} \gamma_{i_{2}}^{2}\left(\frac{1}{2}\left(i_{2}-1\right)^{2} i_{2}+\left(i_{2}-1\right)\left(i_{2}-2\right)\right) \\
& \left.+L_{2-i_{2}} \wedge L_{i_{2}+3}\left(\left(i_{2}-1\right)^{2}-\left(3-i_{2}\right)-\frac{\left(i_{2}-1\right)\left(i_{2}+1\right) i_{2}}{6}\right)\right) \\
\Rightarrow \quad 0= & L_{2-i_{2}^{\prime}} \wedge L_{i_{2}^{\prime}+3}\left(\left(i_{2}^{\prime}-1\right)^{2}-\left(3-i_{2}^{\prime}\right)-\frac{\left(i_{2}^{\prime}-1\right)\left(i_{2}^{\prime}+1\right) i_{2}^{\prime}}{6}\right)
\end{aligned}
$$

and (A.33) implies for $i_{2}^{\prime}>1, \gamma_{i_{2}^{\prime}}^{2} \neq 0$ the solutions $i_{2}^{\prime}=3,4 . i_{2}$ can therefore only take the values $i_{2}=2,3,4$ and one can calculate explicitely that e.g. the term proportional to $L_{1} \wedge L_{5}$ in (A.32) does not vanish so $\gamma_{i_{2}^{\prime}}^{1}=0$. Thus $\delta\left(L_{2}\right)=0$ and iteratively one shows that (3.10) with $m=1$ implies $\delta\left(L_{n}\right)=0$ for $n>2$. For arbitrary positive $m$ one finds

$$
\begin{aligned}
\delta\left(\left[L_{-1}, L_{m}\right]\right) & =-\left[L_{m} \otimes 1+1 \otimes L_{m}, \delta\left(L_{-1}\right)\right] \\
\Rightarrow 0 & =-\sum_{i_{-1} \in I_{-1}} \gamma_{i_{-1}}^{-1}\left(\left(m+1+i_{-1}\right) L_{m-1-i_{-1}} \wedge L_{i_{-1}}+\left(m-i_{-1}\right) L_{-1-i_{-1}} \wedge L_{i_{-1}+m}\right)
\end{aligned}
$$

which yields $\gamma_{i_{-1}^{\prime}}^{-1}=0$ for the largest index $i_{-1}^{\prime}$ and thus $\delta\left(L_{-1}\right)=0$.

Finally, one shows explicitely that (3.10) with $m=1, n=-2$ results in $\delta\left(L_{-2}\right)=0$ and, similarly to the case of positive $m$ that can be used to show that $\delta\left(L_{m}\right)=0$ for all $m<-2$, completing the proof of the first theorem.

\section{A.2 Proof of theorem 2}

Note that a 1-cocycle $\delta$ applied to an element of $\mathfrak{W}$ can be split into three parts $\delta^{I}, \delta^{I I}, \delta^{I I I}$, mapping to $\mathfrak{W} \wedge \mathfrak{W}, \overline{\mathfrak{W}} \wedge \overline{\mathfrak{W}}$ or $\mathfrak{W} \wedge \overline{\mathfrak{W}}$ respectively, which have to satisfy the cocycle condition separately. From the previous theorem it follows that $\delta^{I}$ is cohomolog to zero and from (3.10) one can easily see that $\delta^{I I}$ has to vanish. Thus we only need to consider the part $\delta^{I I I}$ which again can be separated by the degree $d$, which we define such that

$$
\delta\left(L_{m}\right)=L_{i} \wedge \bar{L}_{j}
$$

has $d=i-m$. A general cocycle of homogenous degree $d \neq 0$ is given by

$$
\delta\left(L_{0}\right)=\sum_{j_{0} \in I_{0}} \alpha_{j_{0}}^{0} L_{d} \wedge \bar{L}_{j_{0}}
$$

on $L_{0}$. Setting

$$
r=\sum_{j_{0} \in I_{0}} \frac{\alpha_{j_{0}}^{0}}{d} L_{d} \wedge \bar{L}_{j_{0}}
$$


we then have

$$
\delta^{\prime}\left(L_{0}\right)=\delta\left(L_{0}\right)-\delta_{r}\left(L_{0}\right)=0
$$

Using this in

$$
\delta^{\prime}\left(\left[L_{0}, L_{m}\right]\right)=\left[L_{0} \otimes 1+1 \otimes L_{0}, \delta^{\prime}\left(L_{m}\right)\right]-\left[L_{m} \otimes 1+1 \otimes L_{m}, \delta^{\prime}\left(L_{0}\right)\right]
$$

it follows that

$$
-m \delta^{\prime}\left(L_{m}\right)=-(d+m) \delta^{\prime}\left(L_{m}\right) \Rightarrow \delta^{\prime}\left(L_{m}\right)=0
$$

concluding the proof for $d \neq 0$.

A general degree 0 cocycle has the form

$$
\delta\left(L_{m}\right)=\sum_{i_{m} \in I_{m}} \gamma_{i_{m}}^{m} L_{m} \wedge \bar{L}_{i_{m}}
$$

and by choosing

$$
r=\sum_{i_{1} \in I_{1}} \gamma_{i_{1}} L_{0} \wedge \bar{L}_{i_{1}}
$$

it follows that

$$
\delta^{\prime}\left(L_{1}\right)=\delta\left(L_{1}\right)-\delta_{r}\left(L_{1}\right)=0
$$

Then for $m \neq 1$

$$
\begin{aligned}
\delta^{\prime}\left(\left[L_{m}, L_{1}\right]\right) & =-\left[L_{1} \otimes 1+1 \otimes L_{1}, \delta^{\prime}\left(L_{m}\right)\right] \\
\Rightarrow \sum_{i_{m+1} \in I_{m+1}}(m-1) \gamma_{i_{m+1}}^{m+1} L_{m+1} \wedge \bar{L}_{i_{m+1}} & =\sum_{i_{m} \in I_{m}}(m-1) \gamma_{i_{m}}^{m} L_{m+1} \wedge \bar{L}_{i_{m}}
\end{aligned}
$$

and it follows that

$$
\gamma_{i_{m+1}}^{m+1}=\gamma_{i_{m}}^{m}
$$

If $m=0$ in (A.45) we conclude

$$
0=-\sum_{i_{0} \in I_{0}} \gamma_{i_{0}}^{0} L_{1} \wedge \bar{L}_{i_{0}}
$$

and thus $\gamma_{i_{0}}^{0}=0$. Because of (A.47) $\gamma_{i_{m}}^{m}=\gamma_{i_{0}}^{0}$ for $m<0$ and for $m>0$ all coefficients are given by $\gamma_{i_{m}}^{m}=\gamma_{i_{2}}^{2}$. However, from (3.10) with $m=2, n=3$ we find

$$
-\sum_{i_{2} \in I_{2}} \gamma_{i_{2}}^{2} L_{5} \wedge \bar{L}_{i_{2}}=\sum_{i_{2} \in I_{2}} \gamma_{i_{2}}^{2}(-2) L_{5} \wedge \bar{L}_{i_{2}}
$$

and thus $\gamma_{i_{2}}^{2}=0$, concluding the proof. 


\section{B Classification of triangular r-matrices}

First, note that since $\mathfrak{o}(4, \mathbb{C})=\mathfrak{s l}(2) \oplus \overline{\mathfrak{s l}}(2)$ and $\wedge^{2} \mathfrak{o}(4, \mathbb{C})=\mathfrak{s l}(2) \wedge \mathfrak{s l}(2) \oplus \mathfrak{s l}(2) \wedge \overline{\mathfrak{s l}}(2) \oplus$ $\overline{\mathfrak{s l}}(2) \wedge \overline{\mathfrak{s l}}(2)$ each $\mathrm{r}$-matrix can be split according to

$$
r=a+\bar{a}+b, \quad a \in \mathfrak{s l}(2) \wedge \mathfrak{s l}(2), \bar{a} \in \overline{\mathfrak{s l}}(2) \wedge \overline{\mathfrak{s l}}(2), b \in \mathfrak{s l}(2) \wedge \overline{\mathfrak{s} l}(2) .
$$

Starting with a generic

$$
a=\alpha_{+} L_{1} \wedge L_{0}+\alpha_{0} L_{1} \wedge L_{-1}+\alpha_{-} L_{-1} \wedge L_{0}
$$

triangularity $[[a, a]]=0$ enforces

$$
\alpha_{0}^{2}=-\alpha_{+} \alpha_{-}
$$

Using the automorphism (4.10) with $\gamma=\sqrt{-\frac{\alpha_{-}}{\alpha_{+}}}, \epsilon=1$ in the case $\alpha_{0} \neq 0$ and with $\epsilon=-1(+1)$ if $\alpha_{-}=0\left(\alpha_{+}=0\right)$ we find that there are two one-parameter r-matrices in $\mathfrak{s l}(2) \wedge \mathfrak{s l}(2)$

$$
\begin{aligned}
& a_{1}=\alpha\left(L_{1} \wedge L_{0}+L_{1} \wedge L_{-1}-L_{-1} \wedge L_{0}\right), \\
& a_{2}=\alpha L_{1} \wedge L_{0}
\end{aligned}
$$

and similar for $\overline{\mathfrak{s l}}(2) \wedge \overline{\mathfrak{s} l}(2)$

$$
\begin{aligned}
& \bar{a}_{1}=\bar{\alpha}\left(\bar{L}_{1} \wedge \bar{L}_{0}+\bar{L}_{1} \wedge \bar{L}_{-1}-\bar{L}_{-1} \wedge \bar{L}_{0}\right), \\
& \bar{a}_{2}=\bar{\alpha} \bar{L}_{1} \wedge \bar{L}_{0} .
\end{aligned}
$$

For r-matrices that only contain terms of type $b$ one has to demand $[[b, b]]=0$ and the general result (before applying any automorphisms) as obtained in $[37,38]$ reads

$$
\left(\beta_{+} L_{1}+\beta_{0} L_{0}+\beta_{-} L_{-1}\right) \wedge\left(\bar{\beta}_{+} \bar{L}_{1}+\bar{\beta}_{0} \bar{L}_{0}+\bar{\beta}_{-} \bar{L}_{-1}\right) .
$$

Taking into account the automorphisms (4.10), (4.11) one can represent this as eleven r-matrices with up to four parameters

$$
\begin{array}{rlrl}
b_{1} & =\left(\beta L_{1}+\beta_{0} L_{0}+\beta L_{-1}\right) \wedge\left(\bar{\beta} \bar{L}_{1}+\bar{\beta}_{0} \bar{L}_{0}+\bar{\beta} \bar{L}_{-1}\right), \\
b_{2} & =\left(L_{1}+L_{0}\right) \wedge\left(\bar{\beta} \bar{L}_{1}+\bar{\beta}_{0} \bar{L}_{0}+\bar{\beta} \bar{L}_{-1}\right), & \\
b_{3} & =\left(L_{1}+L_{-1}\right) \wedge\left(\bar{\beta} \bar{L}_{1}+\bar{\beta}_{0} \bar{L}_{0}+\bar{\beta} \bar{L}_{-1}\right), & \\
b_{4} & =\beta\left(L_{1}+L_{0}\right) \wedge\left(\bar{L}_{1}+\bar{L}_{0}\right), & b_{5} & =\beta\left(L_{1}+L_{-1}\right) \wedge\left(\bar{L}_{1}+\bar{L}_{-1}\right), \\
b_{6} & =\beta\left(L_{1}+L_{-1}\right) \wedge\left(\bar{L}_{1}+\bar{L}_{0}\right), & & b_{7}=L_{1} \wedge\left(\bar{\beta} \bar{L}_{1}+\bar{\beta}_{0} \bar{L}_{0}+\bar{\beta} \bar{L}_{-1}\right), \\
b_{8} & =L_{1} \wedge \bar{\beta}\left(\bar{L}_{1}+\bar{L}_{0}\right), & b_{9} & =L_{1} \wedge \bar{\beta}\left(\bar{L}_{1}+\bar{L}_{-1}\right), \\
b_{10} & =L_{1} \wedge \bar{L}_{1}, & b_{11} & =L_{1} \wedge \bar{L}_{0} .
\end{array}
$$

When combining $a, \bar{a}$ and $b$ terms there are two different cases, $[[b, b]]=0$ and $[[b, b]]=$ $-2[[b, a]]-2[[b, \bar{a}]] \neq 0$, that will be analysed separately. In the first case (for the moment considering only $a$ terms) one infers $[[b, a]]=0$ and $[[a, a]]=0$. With the general 
ansatz (B.2) for $a$ and

$$
\begin{aligned}
b= & \beta_{1} L_{1} \wedge \bar{L}_{1}+\beta_{2} L_{1} \wedge \bar{L}_{0}+\beta_{3} L_{1} \wedge \bar{L}_{-1} \\
& +\beta_{4} L_{0} \wedge \bar{L}_{1}+\beta_{5} L_{0} \wedge \bar{L}_{0}+\beta_{6} L_{0} \wedge \bar{L}_{-1} \\
& +\beta_{7} L_{-1} \wedge \bar{L}_{1}+\beta_{8} L_{-1} \wedge \bar{L}_{0} \beta_{9} L_{-1} \wedge \bar{L}_{-1}
\end{aligned}
$$

we extract the equations

$$
\begin{aligned}
-2 \beta_{1} \alpha_{0}+\beta_{4} \alpha_{1} & =0, & -\beta_{4} \alpha_{-1}-2 \beta_{7} \alpha_{0}=0, \\
\beta_{1} \alpha_{-1}+\beta_{7} \alpha_{1} & =0, & -2 \beta_{2} \alpha_{0}+\beta_{5} \alpha_{1}=0, \\
-\beta_{5} \alpha_{-1}-2 \beta_{8} \alpha_{0} & =0, & \beta_{2} \alpha_{-1}+\beta_{8} \alpha_{1}=0, \\
-2 \beta_{3} \alpha_{0}+\beta_{6} \alpha_{1} & =0, & -\beta_{6} \alpha_{-1}-2 \beta_{9} \alpha_{0}=0, \\
\beta_{3} \alpha_{-1}+\beta_{9} \alpha_{1} & =0 &
\end{aligned}
$$

from $[[b, a]]=0$. For the coefficients of $a$ triangularity entails (B.3) and for $b$ we additionaly use the automorphisms to bring them in the form (B.9)-(B.15). For $b=b_{1}$, implying

$$
\beta_{1}=\beta_{3}=\beta_{7}=\beta_{9}, \quad \beta_{2}=\beta_{8}, \quad \beta_{4}=\beta_{6},
$$

the equations (B.17)-(B.21) yield

$$
\alpha_{-1}=-\alpha_{1}, \quad \beta_{4}=2 \beta_{1}, \quad \beta_{5}=2 \beta_{2}
$$

resulting in

$$
r=\left(L_{1}+L_{-1}+2 L_{0}\right) \wedge\left(\beta_{1}\left(\bar{L}_{1}+\bar{L}_{-1}\right)+\beta_{2} \bar{L}_{0}\right)+a_{1} .
$$

Similarly for the other r-matrix components of type $b$ one has

$$
\begin{aligned}
r & \equiv b_{2}+a=L_{1} \wedge\left(\beta_{1}\left(\bar{L}_{1}+\bar{L}_{-1}\right)+\beta_{2} \bar{L}_{0}\right)+a_{2}, \\
r & \equiv b_{3}+a=b_{3}+\alpha\left(L_{1}-L_{-1}\right) \wedge L_{0}, \\
r & \equiv b_{4}+a=\beta L_{1} \wedge\left(\bar{L}_{1}+\bar{L}_{0}\right)+a_{2}, \\
r & \equiv b_{4}+a=\beta\left(L_{1}+L_{0}\right) \wedge \bar{L}_{1}+a_{2}, \\
r & \equiv b_{5}+a=b_{5}+\alpha\left(L_{1}-L_{-1}\right) \wedge L_{0}, \\
r & \equiv b_{6}+a=b_{6}+a_{1}, \\
r & \equiv b_{7}+a=L_{1} \wedge \bar{L}_{0}+a_{2}, \\
r & \equiv b_{8}+a=L_{1} \wedge\left(\bar{L}_{1}+\bar{L}_{0}\right)+a_{2}, \\
r & \equiv b_{9}+a=L_{1} \wedge\left(\bar{L}_{1}+\bar{L}_{-1}\right)+a_{2}, \\
r & \equiv b_{10}+a=L_{1} \wedge \bar{L}_{1}+a_{2} .
\end{aligned}
$$

To classify r-matrices of the form $b+\bar{a}$ one can use (4.11) and that the coefficients of $\bar{b}$ with (B.16) are just the transposed coefficients (if they are represented by a $3 \times 3$ matrix) of 
$b$ and a global minus sign. In the symmetric cases $b_{1}, b_{4}, b_{5}, b_{10}$ the results are automorphic to (B.23)-(B.28) with $\varphi^{\prime}$ and for the rest one has

$$
\begin{aligned}
& r=-\bar{b}_{2}+a=\beta\left(L_{1}+L_{-1}+2 L_{0}\right) \wedge\left(\bar{L}_{1}+\bar{L}_{0}\right)+a_{1}, \\
& r=-\bar{b}_{4}+a=\beta\left(L_{1}+L_{-1}+2 L_{0}\right) \wedge\left(\bar{L}_{1}+\bar{L}_{-1}\right)+a_{1}, \\
& r=-\bar{b}_{6}+a=b_{6}+\alpha L_{1} \wedge\left(L_{0}+2 L_{-1}\right), \\
& r=-\bar{b}_{7}+a=L_{1} \wedge\left(\bar{L}_{1}+\bar{L}_{-1}+2 \bar{L}_{0}\right)+\bar{a}_{1} .
\end{aligned}
$$

While the $r$ in (B.24) and (B.35) are solutions of $[[b, a]]=0$ the $a$ part is not triangular so they have to be discarded. Combining the previous results (and explicitely calculating some "overlaps" of the form $[[b, \bar{a}]])$ we find for $b+a+\bar{a}$ the following possibilities

$$
\begin{aligned}
r & \equiv b_{1}+a+\bar{a}=\left(L_{1}+L_{-1}+2 L_{0}\right) \wedge\left(\bar{L}_{1}+\bar{L}_{1}+2 \bar{L}_{0}\right)+a_{1}+\bar{a}_{1}, \\
r & \equiv b_{2}+a+\bar{a}=\beta L_{1} \wedge\left(\bar{L}_{1}+\bar{L}_{-1}+2 \bar{L}_{0}\right)+a_{2}+\bar{a}_{1}, \\
r & \equiv b_{4}+a+\bar{a}=L_{1} \wedge\left(\bar{L}_{1}+\bar{L}_{0}\right)+a_{2}+\bar{a}_{2}, \\
r & \equiv b_{10}+a+\bar{a}=L_{1} \wedge \bar{L}_{1}+a_{2}+\bar{a}_{2} .
\end{aligned}
$$

In the case $[[b, b]] \neq 0$ we again make use of the results found in $[37,38]$. In particular the general solution for the equation

$$
0 \neq[[b, b]]=-2[[b, a]]-2[[b, \bar{a}]]
$$

up to $\operatorname{Aut}(\mathfrak{o}(4, \mathbb{C}))$ has the form

$$
\alpha L_{1} \wedge L_{-1}-\alpha \bar{L}_{1} \wedge \bar{L}_{-1}+b, \quad \alpha L_{1} \wedge L_{0}+\alpha \bar{L}_{1} \wedge \bar{L}_{0}+b^{\prime},
$$

with specific $b, b^{\prime}$ that are not of interest for now. The first r-matrix in (B.42) is quasitriangular with ad-invariant (in $\mathfrak{o}(4, \mathbb{C})$ ) element containing $\Omega=4 \alpha^{2} L_{1} \wedge L_{0} \wedge L_{-1}+\ldots$ and since the solutions of (B.41) up to $\operatorname{Aut}(\mathfrak{W} \oplus \mathfrak{W})$ are in the orbits of $\mathfrak{o}(4, \mathbb{C})$ automorphisms $\varphi$ containing (B.42) we would need

$$
\varphi(\Omega)=4 \alpha^{2} \varphi\left(L_{1} \wedge L_{0} \wedge L_{-1}\right)+\ldots=0
$$

to obtain a triangular solution. This, however, would entail that the matrix of the coefficients of $\varphi$ has determinant zero but then it would not be invertible and thus $\varphi$ no automorphism. Furthermore there can be no $\mathfrak{o}(4, \mathbb{C})$ automorphism that maps the $a$ terms of the second solution of (B.42) to $a_{1}$ because $a_{1}$ can not be written in the form $\left(\alpha_{1} L_{1}+\alpha_{2} L_{0}+\alpha_{3} L_{-1}\right) \wedge\left(\alpha_{1}^{\prime} L_{1}+\alpha_{2}^{\prime} L_{0}+\alpha_{3}^{\prime} L_{-1}\right)$. We conclude that only r-matrices of the form $b+a_{2}+\bar{a}_{2}$ have to be considered. To this end we extract the equations

$$
\begin{array}{rlrl}
\beta_{1} \beta_{5}-\beta_{2} \beta_{4}+\beta_{4} 2 \alpha & =0, & -\beta_{4} \beta_{8}+\beta_{7} \beta_{5} & =0, \\
\beta_{1} \beta_{8}-\beta_{2} \beta_{7}+\beta_{7} 2 \alpha & =0, & 2 \beta_{1} \beta_{6}-2 \beta_{3} \beta_{4}+\beta_{5} 2 \alpha & =0, \\
-2 \beta_{4} \beta_{9}+2 \beta_{6} \beta_{7} & =0, & 2 \beta_{1} \beta_{9}-2 \beta_{3} \beta_{7}+\beta_{8} 2 \alpha & =0, \\
-\beta_{3} \beta_{5}+\beta_{2} \beta_{6}+\beta_{6} 2 \alpha & =0, & -\beta_{5} \beta_{9}+\beta_{6} \beta_{8} & =0, \\
-\beta_{3} \beta_{8}+\beta_{2} \beta_{9}+\beta_{9} 2 \alpha & =0 &
\end{array}
$$


from (B.41). Additionaly we also get the same eqations with $\alpha \rightarrow-\bar{\alpha}$ and in the terms proportional to $\bar{\alpha}$ the coefficients of $b$ are transposed. Solving these equations yields only the solution

$$
\beta_{1} L_{1} \wedge \bar{L}_{1}+\beta_{2}\left(L_{1} \wedge \bar{L}_{0}+\bar{L}_{1} \wedge L_{0}\right)+\beta_{2} L_{1} \wedge L_{0}+\beta_{2} \bar{L}_{1} \wedge \bar{L}_{0}
$$

i.e. the same as in $[37,38]$.

After removing duplicacies all the r-matrices we found can be casted into the classes (4.12)-(4.19).

Open Access. This article is distributed under the terms of the Creative Commons Attribution License (CC-BY 4.0), which permits any use, distribution and reproduction in any medium, provided the original author(s) and source are credited.

\section{References}

[1] A. Staruszkiewicz, Gravitation Theory in Three-Dimensional Space, Acta Phys. Polon. 24 (1963) 735 [INSPIRE].

[2] S. Deser, R. Jackiw and G. 't Hooft, Three-Dimensional Einstein Gravity: Dynamics of Flat Space, Annals Phys. 152 (1984) 220 [INSPIRE].

[3] S. Deser and R. Jackiw, Three-Dimensional Cosmological Gravity: Dynamics of Constant Curvature, Annals Phys. 153 (1984) 405 [INSPIRE].

[4] S. Carlip, Quantum gravity in $2+1$ dimensions, Cambridge University Press, Cambridge U.K. (1998).

[5] E. Witten, (2+1)-Dimensional Gravity as an Exactly Soluble System, Nucl. Phys. B 311 (1988) 46 [INSPIRE].

[6] A. Achucarro and P.K. Townsend, A Chern-Simons Action for Three-Dimensional anti-de Sitter Supergravity Theories, Phys. Lett. B 180 (1986) 89 [InSPIRE].

[7] J.D. Brown and M. Henneaux, Central Charges in the Canonical Realization of Asymptotic Symmetries: An Example from Three-Dimensional Gravity, Commun. Math. Phys. 104 (1986) 207 [INSPIRE].

[8] J.M. Maldacena, The Large $N$ limit of superconformal field theories and supergravity, Adv. Theor. Math. Phys. 2 (1998) 231 [hep-th/9711200] [INSPIRE].

[9] P. Kraus, Lectures on black holes and the AdS $S_{3} / C F T_{2}$ correspondence, Lect. Notes Phys. 755 (2008) 193 [hep-th/0609074] [INSPIRE].

[10] L. Freidel, Reconstructing AdS/CFT, arXiv:0804.0632 [INSPIRE].

[11] M. Bañados, C. Teitelboim and J. Zanelli, The Black hole in three-dimensional space-time, Phys. Rev. Lett. 69 (1992) 1849 [hep-th/9204099] [INSPIRE].

[12] M. Bañados, M. Henneaux, C. Teitelboim and J. Zanelli, Geometry of the $(2+1)$ black hole, Phys. Rev. D 48 (1993) 1506 [Erratum ibid. 88 (2013) 069902] [gr-qc/9302012] [INSPIRE].

[13] S. Carlip, Conformal field theory, $(2+1)$-dimensional gravity, and the BTZ black hole, Class. Quant. Grav. 22 (2005) R85 [gr-qc/0503022] [inSPIRE]. 
[14] E. Witten, Quantum Field Theory and the Jones Polynomial, Commun. Math. Phys. 121 (1989) 351 [INSPIRE].

[15] V.V. Fock and A.A. Rosly, Poisson structure on moduli of flat connections on Riemann surfaces and r matrix, Am. Math. Soc. Transl. 191 (1999) 67 [math/9802054] [INSPIRE].

[16] A.Y. Alekseev and A.Z. Malkin, Symplectic structures associated to Lie-Poisson groups, Commun. Math. Phys. 162 (1994) 147 [hep-th/9303038] [INSPIRE].

[17] A.Y. Alekseev and A.Z. Malkin, Symplectic structure of the moduli space of flat connection on a Riemann surface, Commun. Math. Phys. 169 (1995) 99 [hep-th/9312004] [InSPIRE].

[18] F.A. Bais, N.M. Muller and B.J. Schroers, Quantum group symmetry and particle scattering in (2+1)-dimensional quantum gravity, Nucl. Phys. B 640 (2002) 3 [hep-th/0205021] [INSPIRE].

[19] C. Meusburger and B.J. Schroers, Poisson structure and symmetry in the Chern-Simons formulation of $(2+1)$-dimensional gravity, Class. Quant. Grav. 20 (2003) 2193 [gr-qc/0301108] [INSPIRE].

[20] C. Meusburger and B.J. Schroers, The quantisation of Poisson structures arising inChern-Simons theory with gauge group $G \ltimes \mathfrak{g}^{*}$, Adv. Theor. Math. Phys. 7 (2003) 1003 [hep-th/0310218] [INSPIRE].

[21] C. Meusburger and B.J. Schroers, Quaternionic and Poisson-Lie structures in 3d gravity: The Cosmological constant as deformation parameter, J. Math. Phys. 49 (2008) 083510 [arXiv: 0708.1507] [INSPIRE].

[22] L. Freidel and E.R. Livine, Ponzano-Regge model revisited III: Feynman diagrams and effective field theory, Class. Quant. Grav. 23 (2006) 2021 [hep-th/0502106] [INSPIRE].

[23] L. Freidel and E.R. Livine, 3D Quantum Gravity and Effective Noncommutative Quantum Field Theory, Phys. Rev. Lett. 96 (2006) 221301 [hep-th/0512113] [InSPIRE].

[24] H. Bondi, M.G.J. van der Burg and A.W.K. Metzner, Gravitational waves in general relativity. 7. Waves from axisymmetric isolated systems, Proc. Roy. Soc. Lond. A 269 (1962) 21 [INSPIRE].

[25] R.K. Sachs, Gravitational waves in general relativity. 8. Waves in asymptotically flat space-times, Proc. Roy. Soc. Lond. A 270 (1962) 103 [InSPIRE].

[26] R. Sachs, Asymptotic symmetries in gravitational theory, Phys. Rev. 128 (1962) 2851 [INSPIRE].

[27] A. Borowiec, L. Brocki, J. Kowalski-Glikman and J. Unger, $\kappa$-deformed BMS symmetry, Phys. Lett. B 790 (2019) 415 [arXiv:1811.05360] [INSPIRE].

[28] A. Borowiec, L. Brocki, J. Kowalski-Glikman and J. Unger, BMS algebras in 4 and 3 dimensions, their quantum deformations and duals, JHEP 02 (2021) 084 [arXiv:2010.10224] [INSPIRE].

[29] M. Campiglia and A. Laddha, Asymptotic symmetries and subleading soft graviton theorem, Phys. Rev. D 90 (2014) 124028 [arXiv:1408.2228] [INSPIRE].

[30] F. Cianfrani, J. Kowalski-Glikman, D. Pranzetti and G. Rosati, Symmetries of quantum spacetime in three dimensions, Phys. Rev. D 94 (2016) 084044 [arXiv:1606.03085] [INSPIRE]. 
[31] J. Lukierski, H. Ruegg, A. Nowicki and V.N. Tolstoi, Q deformation of Poincaré algebra, Phys. Lett. B 264 (1991) 331 [INSPIRE].

[32] J. Lukierski, A. Nowicki and H. Ruegg, New quantum Poincaré algebra and $k$ deformed field theory, Phys. Lett. B 293 (1992) 344 [InSPIRE].

[33] J. Lukierski, H. Ruegg and W.J. Zakrzewski, Classical quantum mechanics of free kappa relativistic systems, Annals Phys. 243 (1995) 90 [hep-th/9312153] [INSPIRE].

[34] S. Majid and H. Ruegg, Bicrossproduct structure of kappa Poincaré group and noncommutative geometry, Phys. Lett. B 334 (1994) 348 [hep-th/9405107] [INSPIRE].

[35] A. Borowiec, J. Lukierski and V.N. Tolstoy, Basic quantizations of D $=4$ Euclidean, Lorentz, Kleinian and quaternionic $\mathfrak{o}^{\star}(4)$ symmetries, JHEP 11 (2017) 187 [arXiv:1708.09848] [INSPIRE].

[36] S.-H. Ng and E.J. Taft, Classification of the Lie bialgebra structures on the Witt and Virasoro algebras, J. Pure Appl. Algebra 151 (2000) 67.

[37] A. Borowiec, J. Lukierski and V.N. Tolstoy, Quantum deformations of $D=4$ Euclidean, Lorentz, Kleinian and quaternionic $\mathfrak{o}^{*}(4)$ symmetries in unified $\mathfrak{o}(4 ; \mathbb{C})$ setting, Phys. Lett. $B$ 754 (2016) 176 [arXiv:1511.03653] [INSPIRE].

[38] A. Borowiec, J. Lukierski and V.N. Tolstoy, Addendum to: "Quantum deformations of $D=4$ Euclidean, Lorentz, Kleinian and quaternionic $\mathfrak{o}^{*}(4)$ symmetries in unified $\mathfrak{o}(4 ; \mathbb{C})$ setting", Phys. Lett. B 770 (2017) 426 [arXiv:1704.06852] [INSPIRE].

[39] A. Strominger, Lectures on the Infrared Structure of Gravity and Gauge Theory, arXiv: 1703.05448 [INSPIRE].

[40] J. Kowalski-Glikman, J. Lukierski and T. Trześniewski, Quantum D $=3$ Euclidean and Poincaré symmetries from contraction limits, JHEP 09 (2020) 096 [arXiv:1911.09538] [INSPIRE].

[41] H.R. Safari and M.M. Sheikh-Jabbari, BMS 4 algebra, its stability and deformations, JHEP 04 (2019) 068 [arXiv: 1902.03260] [INSPIRE].

[42] M. Enriquez-Rojo, T. Procházka and I. Sachs, On deformations and extensions of Diff( $\left.S^{2}\right)$, JHEP 10 (2021) 133 [arXiv:2105.13375] [InSPIRE].

[43] G. Compère, A. Fiorucci and R. Ruzziconi, The $\Lambda$-BMS $S_{4}$ group of $d S_{4}$ and new boundary conditions for AdS $S_{4}$, Class. Quant. Grav. 36 (2019) 195017 [Erratum ibid. 38 (2021) 229501] [arXiv: 1905.00971] [INSPIRE].

[44] G. Compère, A. Fiorucci and R. Ruzziconi, The $\Lambda$-BMS 4 charge algebra, JHEP 10 (2020) 205 [arXiv: 2004.10769] [INSPIRE].

[45] B. Oblak, BMS Particles in Three Dimensions, Ph.D. Thesis, Brussels University, Brussels Belgium (2016) [arXiv: 1610.08526] [INSPIRE].

[46] G. Compère and A. Fiorucci, Advanced Lectures on General Relativity, arXiv:1801.07064 [INSPIRE].

[47] V. Chari and A. Pressley, A Guide to Quantum Groups, Cambridge University Press, Cambridge U.K. (1995) [ISBN:978-0521558846].

[48] A. Klimyk and K. Schmüdgen, Quantum Groups and Their Representations, Springer, Heidelberg Germany (2011) [ISBN:978-3642646010]. 
[49] P.I. Etingof and O. Schiffmann, Lectures on Quantum Groups, International Press of Boston, Inc., Boston U.S.A. (2010) [ISBN:978-1571462077].

[50] J. Ecker and M. Schlichenmaier, The low-dimensional algebraic cohomology of the Witt and the Virasoro algebra, J. Phys. Conf. Ser. 1194 (2019) 012032 [inSPIRE].

[51] P. Stachura, Poisson-Lie structures on Poincaré and Euclidean groups in three dimensions, J. Phys. A 31 (1998) 4555.

[52] A. Borowiec and A. Pachol, $\kappa$-Deformations and Extended $\kappa$-Minkowski Spacetimes, SIGMA 10 (2014) 107 [arXiv: 1404.2916] [INSPIRE].

[53] R. Penrose, The geometry of impulsive gravitational waves, in General Relativity, Papers in Honour of J.L. Synge, Clarendon Press, Oxford U.K. (1972), pg. 101.

[54] A. Strominger and A. Zhiboedov, Superrotations and Black Hole Pair Creation, Class. Quant. Grav. 34 (2017) 064002 [arXiv: 1610.00639] [INSPIRE].

[55] R. Gleiser and J. Pullin, Are cosmic strings gravitationally stable topological defects?, Class. Quant. Grav. 6 (1989) L141 [InSPIRE].

[56] L. Junbo, S. Yucai and X. Bin, Lie Bialgebra Structures on the Centerless W-Algebra W(2,2), Algebra Colloq. 17 (2010) 181.

[57] G. Compère, W. Song and A. Strominger, New Boundary Conditions for AdS3, JHEP 05 (2013) 152 [arXiv: 1303.2662] [INSPIRE].

[58] O. Fuentealba, H.A. González, A. Pérez, D. Tempo and R. Troncoso, Superconformal Bondi-Metzner-Sachs Algebra in Three Dimensions, Phys. Rev. Lett. 126 (2021) 091602 [arXiv: 2011.08197] [INSPIRE].

[59] H. Afshar et al., Soft Heisenberg hair on black holes in three dimensions, Phys. Rev. D 93 (2016) 101503 [arXiv:1603.04824] [INSPIRE].

[60] D. Grumiller, A. Pérez, M.M. Sheikh-Jabbari, R. Troncoso and C. Zwikel, Spacetime structure near generic horizons and soft hair, Phys. Rev. Lett. 124 (2020) 041601 [arXiv: 1908.09833] [INSPIRE].

[61] C. Batlle, V. Campello and J. Gomis, A canonical realization of the Weyl BMS symmetry, Phys. Lett. B 811 (2020) 135920 [arXiv:2008.10290] [INSPIRE].

[62] H. Adami, M.M. Sheikh-Jabbari, V. Taghiloo, H. Yavartanoo and C. Zwikel, Symmetries at null boundaries: two and three dimensional gravity cases, JHEP 10 (2020) 107 [arXiv:2007.12759] [INSPIRE].

[63] G. Barnich, Centrally extended BMS4 Lie algebroid, JHEP 06 (2017) 007 [arXiv: 1703.08704] [INSPIRE].

[64] J.H. Lu, Hopf algebroids and quantum groupoids, Int. J. Math. 7 (1996) 47 [q-alg/9505024].

[65] T. Brzezinski and G. Militaru, Bialgebroids, $\times_{A}$-Bialgebras and Duality, J. Algebra 251 (2002) 279.

[66] A. Borowiec and A. Pachoł, Twisted bialgebroids versus bialgebroids from a Drinfeld twist, J. Phys. A 50 (2017) 055205 [arXiv: 1603.09280] [INSPIRE]. 